\title{
Rapid Variations of Earth's Core Magnetic Field
}

\author{
V. Lesur ${ }^{1} \cdot$ N. Gillet $^{2} \cdot$ M. D. Hammer ${ }^{3} \cdot$ M. Mandea ${ }^{4}$
}

Received: 9 March 2021 / Accepted: 19 July 2021 / Published online: 6 January 2022

(c) The Author(s) 2022

\begin{abstract}
Evidence of fast variations in the Earth's core field are seen both in magnetic observatory and satellite records. We present here how they have been identified at the Earth's surface from ground-based observatory records and how their spatio-temporal structure is now characterised by satellite data. It is shown how their properties at the core mantle boundary are extracted through localised and global modelling processes, paying particular attention to their time scales. Finally are listed possible types of waves in the liquid outer core, together with their main properties, that may give rise to these observed fast variations.
\end{abstract}

Keywords Geomagnetism · Magnetic field modelling · Core time scales

\section{Article Highlights}

- The evidence for rapid variations of the core magnetic field is presented and discussed

- The limitations of current core magnetic field models, and how these affect core time scales, are identified

- An overview is given of possible core processes that are linked to fast magnetic field variations in the core

M. Mandea

Mioara.Mandea@cnes.fr

V. Lesur

lesur@ipgpg.fr

1 Université de Paris, Institut de physique du globe de Paris, CNRS, 75005 Paris, France

2 Univ. Grenoble Alpes, Univ. Savoie Mont Blanc, CNRS, IRD, UGE, ISTerre, 38000 Grenoble, France

3 Division of Geomagnetism and Geospace, DTU Space, Technical University of Denmark, Centrifugevej 365, 2800 Kongens Lyngby, Denmark

4 CNES - Centre National d'Etudes Spatiales, 2 place Maurice Quentin, 75039 Paris Cedex 01, France 


\section{Introduction}

The observed magnetic field above the Earth's surface results from the contribution of numerous sources situated inside the Earth, such as the core and the lithosphere, or outside the Earth, such as the ionosphere and magnetosphere. The dominant contribution is by far the field generated in the core. Its longer time-changes, including millennial and longer periods, are reconstructed from paleomagnetism (Constable and Johnson 2005). The shortest fluctuations likely reach sub-daily periods, in link with the rotating fluid dynamics of the fluid core. However, the variations directly observable at the Earth's surface are on a limited range of time scales. The upper limit is slightly longer than a century, corresponding to the installation of the first magnetic observatories (e.g., Matzka et al. 2010). The lower limit is poorly known for two reasons. Firstly, the amplitude of the core signal gets weaker towards high frequencies, so that external signals dominate the magnetic records on periods shorter than $O(1)$ year. Secondly, the screening effect of the conductive mantle, which filters out rapid signals originating from the core, is not well estimated. The aim of this paper is to review our current knowledge on the rapid variations of the core field and the possible core flow perturbations that generate them.

Very early in the study of the Earth's magnetism, it had been realised that the magnetic field $\mathbf{B}$ is changing with time (e.g., Kono 2007). The proper description of this evolution came with the setting of magnetic observatories from the middle of the nineteenth century onward. The general assumption was that most of observed fast variations in $\mathbf{B}(t)$ were due to external fields perturbations, while the dominant core field varied slowly over time. The accumulation of long time series of magnetic observations led to an evolution of this paradigm since, at observatory sites, the main field secular variation ( $\mathrm{SV}$, or rate of change of the field, $\partial \mathbf{B} / \partial t$ ) often appeared as linear trends with abrupt changes of slopes. These singular events are clearly generated in the core and have been called "geomagnetic jerks" (Courtillot et al. 1978). Early discussions on the origin of these events can be found in Malin and Hodder (1982) and Alldredge (1984). The evolution of this secular variation has also been studied in terms of secular acceleration (SA, or second time derivative, $\partial^{2} \mathbf{B} / \partial t^{2}$ ). Early models of the SA can be found in Cain et al. (1967), who used OGO satellite data, assuming the acceleration constant over time. The acceleration was also assumed constant by Malin (1969) and Barraclough and Malin (1979), although in these latter publications it is already noticed that the difference of SA obtained by different authors likely reveals a temporal change. After the MAGSAT satellite mission in 1980, came the first robust representations of magnetospheric fields (Langel and Estes 1985a, b), as well as temporal parameterisations of the core field changes using B-splines (Langel et al. 1986; Bloxham and Jackson 1992; Sabaka et al. 1997). In principle, such models are able to describe the SA and its evolution, but these are not discussed in the aforementioned publications. After the launch of the Oersted and CHAMP satellite missions, it became possible to derive more accurate magnetic field models. While preparing candidate models for the 10th IGRF generation (International Geomagnetic Reference Field, Maus et al. 2005a), it became obvious that assuming a linear evolution of the magnetic field was not adequate to fit the few years of available satellite data: including an acceleration was necessary (Lesur et al. 2005; Olsen et al. 2005b; Maus et al. 2005c). Soon after came models fitting these data and with a temporal description of the core field using B-splines, initially of order four as in the first versions of the CHAOS model (Olsen et al. 2006; Olsen and Mandea 2008), and then five in the GRIMM model (Lesur et al. 2008). With this latter model, it was possible to follow the SA variations over 5 years and to identify a maximum of acceleration in 2006 . From there on, 
numerous publications (e.g., Chulliat and Maus 2014; Finlay et al. 2016) have focused on the acceleration patterns, highlighting pulses in the SA norm at inter-annual periods. These are discussed in the following of this paper.

The crucial issue regarding field models derived from magnetic data is their temporal resolution. Observatory time series show a temporal spectrum $S(f) \propto f^{\alpha}$ with a slope $\alpha \simeq-4$ in the range of periods from $\approx 70$ yrs down to a couple of years (De Santis et al. 2003). This property, which is coherent with the existence of geomagnetic jerks, is also recovered in time series of the geomagnetic Gauss coefficients (Lesur et al. 2018). It is shared by the family of stochastic processes (of order 2) that is differentiable only once in time (Gillet et al. 2013). For those, the estimate of the SA is subject to the choice of sampling rate. With most field models, an instantaneous measure of the SA is not available, but instead only a filtered vision. This time resolution strongly depends on the spherical harmonic degree: the larger the length-scale, the better the resolution in frequency (see Sect. 3.2). A magnetic signal probably exists at inter-annual periods, which carries some information about the core physics. However, the image of the shorter wavelengths is limited to long periods, which alters our reconstruction of inter-annual SA patterns. This limitation precludes the identification of the cutting frequency over which the slope $S(f) \propto f^{-4}$ stops. This depends on the one hand on the conductance of the mantle (Jault 2015) and on the other hand on the relative importance of diffusion and propagation of Alfvén waves in the fluid outer core (Aubert and Gillet 2021).

There is a general agreement that the flow pattern in the liquid outer core that generates most of the observed SV (by advection of the core field) is a large gyre, that flows westwards near the core mantle boundary under Africa and the Atlantic, and flows closer to the cylinder aligned with the rotation axis and tangent to the inner core under the Pacific Ocean (e.g., Pais and Jault 2008; Gillet et al. 2009, 2015; Aubert 2014; Baerenzung et al. 2018). It has been demonstrated, however, that this flow has to vary in time to explain the observed magnetic field variations at observatory sites (Bloxham 1992; Waddington et al. 1995). There are some indications that these flow variations are mainly located under Eastern Asia, and Central America (e.g., Finlay et al. 2016), but they have also been identified close to the inner core tangent cylinder (Livermore et al. 2017; Gillet et al. 2019). Torsional waves with a periodicity around 6 years have also been identified in flow models derived from ground-based observations (Gillet et al. 2010). They explain an independent 6-yr signal recorded in the length-of-day (Abarca del Rio et al. 2000; Holme and De Viron 2013; Chao et al. 2014), and their physics determine the intensity of the magnetic field within the core (where the potential field approximation drops due to electrical currents). The magnetic signature of these waves at Earth's surface is tiny [a couple of $n \mathrm{~T} \mathrm{yr}^{-1}$ - e.g., Cox et al. (2016)] and must be coherent within the set of observation sites in order to imprint core flow models. This magnitude is nevertheless too weak to entirely explain inter-annual $\mathrm{SV}$ changes (of the order of $8 \mathrm{nT} \mathrm{yr}^{-1}$ ), which thus require the existence of other, nonzonal, transient motions (Gillet et al. 2015; Finlay et al. 2016; Kloss and Finlay 2019).

The remainder of this paper is organised as follows: In Sect. 2, we first come back to long series of observatory data and subsequently satellite (geomagnetic virtual observatory, GVO) data, from which we discuss the observed SV evolutions over time. We present next in Sect. 3 different modelling techniques and evaluate how these affect the derived acceleration time scales. Then, possible flow structures and waves that may generate observable signals at Earth surface are reviewed in Sect. 4. We summarise our conclusions in Sect. 5. 


\section{Core Field Variability Observed from Magnetic Data}

\subsection{Ground Magnetic Observatories}

A strong motivation in running magnetic observatories is the monitoring of the Earth's main magnetic field evolution originating in the fluid outer core. The importance of long-term observations in support of this endeavour is illustrated in Fig. 1 where (irregular) declination measurements at a number of locations in the vicinity of Paris, and regular measurements at the observatories in Saint Maur des Fossés (Val de Marne, France, 1883-1902), Val Joyeux (Yvelines, France, 1903-1935) and Chambon-la-Forêt (1936-present) are plotted together. The Paris declination series starts as early as in the sixteenth century, with the first measurement performed in 1541 , giving a value of $7^{\circ}$ E. Let us underline that all declination measurements made in the Paris area (Issy-lesMoulineaux, Montsouris et Montmorency) and in previous French magnetic observatories are reduced to the current location of Chambon-la-Forêt. A detailed discussion of the applied corrections is given in Alexandrescu et al. (1996). The plot shows that, over the last 479 years, the magnetic declination has varied between extreme values reaching $13^{\circ} 30^{\prime} \mathrm{E}$ in 1580 to $22^{\circ} 30^{\prime} \mathrm{W}$ in 1814 . Long data series, such as those in Fig. 1, have been compiled for example for London, Rome and Munich by Malin and Bullard (1981), Cafarella et al. (1992) and Korte et al. (2009), respectively. These series are important in characterising the scales of temporal variations in the core-generated field.

These unique series allow us easily to compute the SV in a single position and to estimate occurrence dates for geomagnetic jerks, mainly before the twentieth century. In Fig. 1, the SV is computed as annual mean differences. To enhance rapid events and reduce solar cycle effects, the first time derivative is computed after applying an 11-yr smoothing low-pass filter window which makes the changes in the trend more rounded. Nevertheless, the geomagnetic jerks can still be clearly identified. The figure clearly shows that, prior to the twentieth century, one of the most prominent geomagnetic jerks appears around 1870. This event is also observed in four other European locations (Alexandrescu et al. 1997) and has recently been detected in the Munich curve (Korte et al. 2009), although a few years earlier. Going farther back in time, there is evidence of changes in the secular variation trend, supported by measurements around epochs $1600,1665,1700,1730,1750,1760,1770,1810,1870,1890$, and 1900. These dates

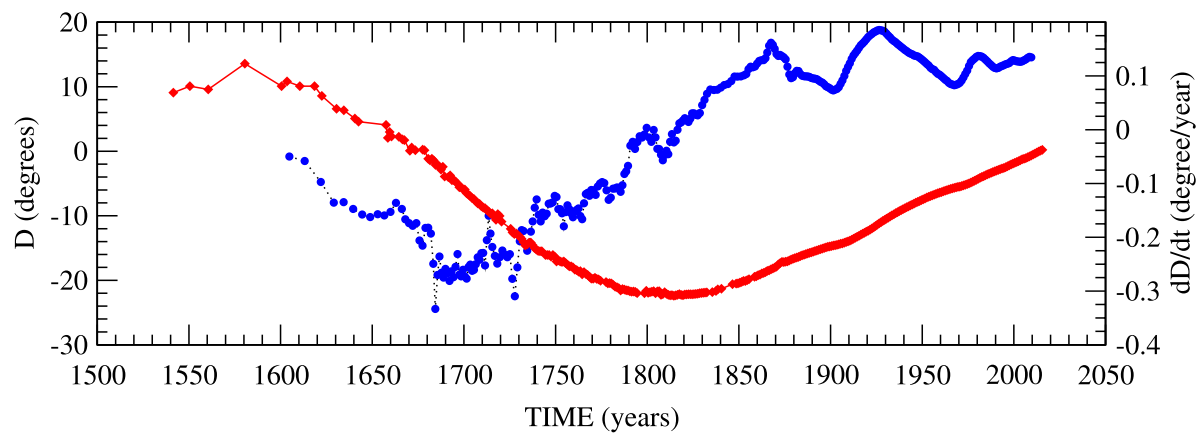

Fig. 1 Compiled records of the observed declination ( $D$, in red) and its estimated secular variation (SV, in blue) in the vicinity of Paris, France. All records have been reduced to a single position at the current site of the French national magnetic observatory in Chambon-la-Forêt (CLF-Loiret, France) 
are close to those detected by Qamili et al. (2013): 1603, 1663, 1703, 1733, 1751, 1763, $1770,1810,1868,1870,1888$, and 1900, when analysing the temporal behaviour of the difference between predicted and actual geomagnetic field model values for successive intervals from 1600 to 1980 , based on the GUFM1 geomagnetic model (Jackson et al. 2000).

The typical SV time scale spans years to centuries, and its typical magnitude is 10-100 $\mathrm{nT} \mathrm{yr}^{-1}$. These values help to define the observational requirements for magnetic observatories: they should run for many years, achieve measurement accuracy in all components of the field vector of around $1 \mathrm{nT}$, and maintain the long-term stability needed to resolve the typical secular change signal. With the above recommendation, the geomagnetic observatories represent a unique dataset to understand the secular variation over long-term periods. Indeed, the first geomagnetic jerk that was detected was identified in geomagnetic observatories series (Courtillot et al. 1978). In this pioneering paper, it was defined as an abrupt turning point separating the otherwise linear trends of the East component of SV prior to and after 1970 at several northern hemisphere observatories.

The geomagnetic jerks can be observed in the various field derivatives (core field, secular variation and secular acceleration) with a wide variety of detection methods. A detection method needs to consider several factors linked to the available data: noise content in the data and its origin, the asynchronous form of a jerk in each field component, temporal and spatial scales at which an event is significant enough to be seen as a jerk. These factors are briefly discussed here:

- the quality of data varies back in time. Systematic geomagnetic field observations at multiple locations, for the three field components, began with the establishment, by Gauss, of the Göttingen Magnetic Union in 1834. Before that date only directional observations were available, so the characterisation of the secular variation via the full vector field was not possible. Only over the twentieth century, the three field components are available for a set of observatories allowing comparison between different locations and different field components.

- the first data analyses were focused on the East component, mainly for two reasons. The first analyses considered mainly European observatories and the geomagnetic jerks are generally clearly noted in this component time series (see Fig. 2). It should be noted that the behaviour of the East component secular variation is different for CLF $\left(48.025^{\circ} \mathrm{N}, 2.260^{\circ} \mathrm{E}\right)$ and $\mathrm{NGK}\left(52.07^{\circ} \mathrm{N}, 12.68^{\circ} \mathrm{E}\right)$ observatories, even if these two observatories are relatively close.

- the magnetic field Eastern component is less affected by the external sources, i.e. electrical currents flowing in the ionosphere and magnetosphere of the Earth. Different methods have been applied to minimise the external field contributions in geomagnetic series (monthly or annual means), via geomagnetic indices or a combination of field models and magnetic indices, or empirical techniques (e.g., De Michelis et al. 2000; Verbanac et al. 2007; Wardinski and Holme 2011). Different attempts have been also applied to parameterise the external field sources during the modelling processes (see Sect. 3.2 and references there in). These models are also suitable to obtain series of data and to investigate geomagnetic jerks, mainly when considering three field components.

- it is well known that the geomagnetic observatories are located mainly on continental areas and that the northern hemisphere is better covered than the southern hemisphere. It is then important to recall the effect of the uneven distribution of observatories in 


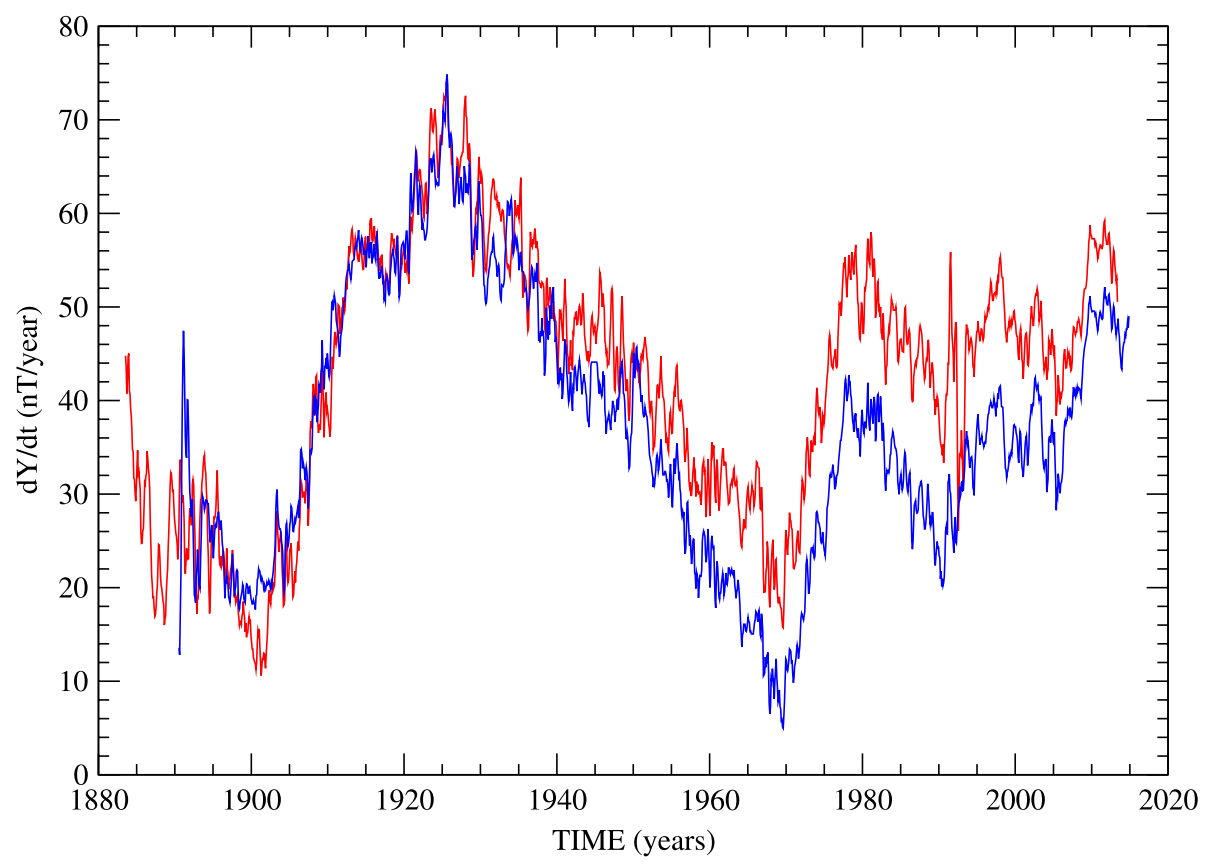

Fig. 2 In red is shown the secular variation for the East component at Chambon-la-Forêt (CLF- $48.025^{\circ} \mathrm{N}$, $\left.2.260^{\circ} \mathrm{E}\right)$, France and, in blue, at Niemegk (NGK-52.07 $\left.\mathrm{N}, 12.68^{\circ} \mathrm{E}\right)$, Germany

the definition of geomagnetic jerk characteristics. Identifying regional jerks has been possible not only because more and more sophisticated methods have been applied (see Mandea et al. 2010; Brown et al. 2013), but also due to the usage of full field geometry.

Although geomagnetic jerks are clearly of internal origin and are well-identified events in magnetic observatories time series, they are less and less seen as singular events in terms of flow dynamics. Study of recent magnetic field models and progresses in numerical geodynamo models suggest that they are natural features of the magnetic field generation in the Earth's core (see Sect. 4).

In order to get a better understanding of the recorded signal variability at magnetic observatories, in Fig. 3 are presented the time series of the three components of the magnetic field in an Earth-Centred Earth Fixed system of coordinates (ECEF-i.e. $Z$ is the Earth's rotation axis, $X$ points towards Greenwich meridian and $Y$ complete this system), together with their Fourier spectra. Data are daily means. The time series have been corrected for recorded jumps, interpolated for missing values using smoothed cubic-splines and "detrended" by subtracting a linear polynomial going through the first and last point of the series. Figure 3 thus displays deviations from a linear evolution of the recorded data. All plots have the same scales, and deviations are much larger in Hermanus observatory (HER, $-34.425^{\circ} \mathrm{N}, 19.225^{\circ} \mathrm{E}$, South-Africa) than on M'bour and Chambon-la-Forêt observatories, $\left(\mathrm{MBO}, 14.392^{\circ} \mathrm{N},-16.958^{\circ} \mathrm{E}\right.$, Senegal) and (CLF, $48.025^{\circ} \mathrm{N}, 2.260^{\circ} \mathrm{E}$, France), respectively. Because of the choice of coordinate system, a large part of the signals generated in the magnetosphere is concentrated in the $Z$ component. These signals are associated in the Fourier spectra with peaks at 1 year and half-year periods. They are also related to excess of energy around 27-day periods and other sub-harmonics. The 11-yr solar cycle 

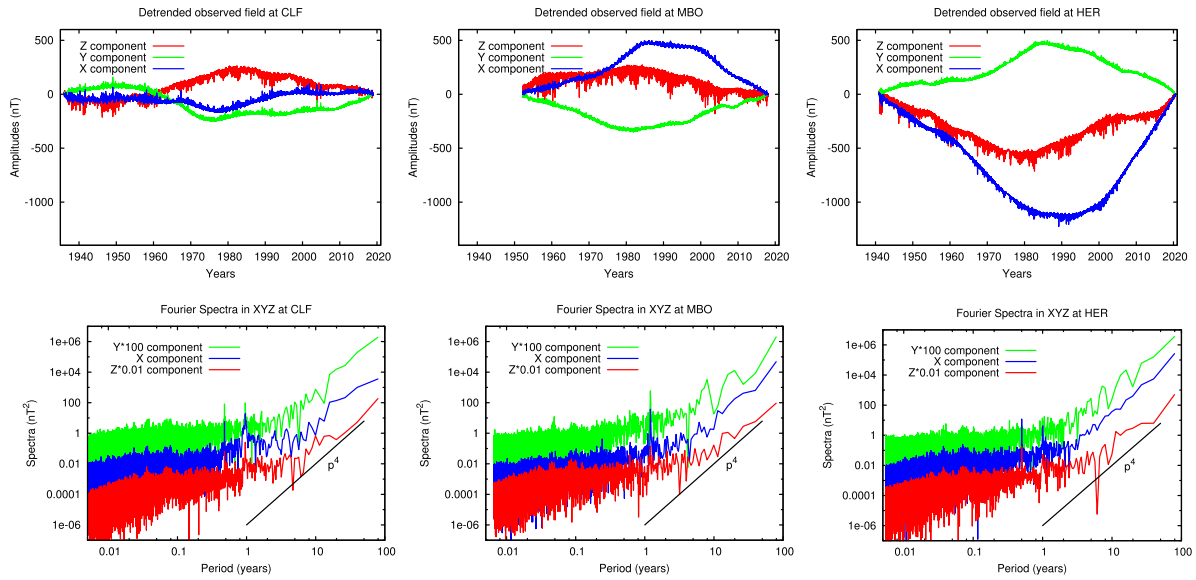

Fig. 3 First row: detrended three components of the magnetic field recorded at observatory sites in ECEF system of coordinates. Second row: Fourier spectra of the corresponding time series where $Y$ and $Z$ components have been shifted up and down, respectively, for clarity. The three presented observatories are from left to right: CLF, MBO, HER (see text for details). The slope $\propto p^{4}$, with $p$ the period, is indicated with a black line

generates small peaks in $Z$ components at all observatories. However, it is extremely difficult to identify specific periodicities for signals generated in the core, as the external field contributions dominate the spectra up to periods as large as 2 to 5 years, depending on locations and components. There is a clear excess in energy at M'bour in the $Y$ component for periods centred on 6.5 years and also for periods from 13 to 15 years. Less distinctive are these same periods for Hermanus observatory. However, at Chambon-la-Forêt, in spite of a very long series starting the 1st January 1936, these periods do not dominate the spectra. The only unexpected maximum is at 1.7 years periods in the $Y$ component. All observatories at the longest periods present an increasing trend that, as expected, roughly correspond to a $p^{4}$ behaviour, with $p$ the period. However, these observations indicate clearly the difference in the content of the observatory records depending on their regions.

\subsection{Virtual Observatories}

With the advent of magnetic field measurements from low Earth orbiting satellites, smaller spatial structures of the core field changes can be mapped due to almost complete global coverage. The last 20 years of continuous space records of the magnetic field provide valuable insights into the field and how it is changing in space and time. In particular, magnetic field measurements from the Danish Oersted satellite (1999-2014), the German CHAMP satellite (2000-2010) and the European Swarm satellite trio mission (2013-), have dramatically increased the constraint from geomagnetic data (Olsen and Stolle 2012; Domingos et al. 2019). In addition to these, calibrated CryoSat-2 satellite measurements (2010-) can be used to generate further constraints on the field variations, in particular during the temporal gap 2010-2014 between CHAMP and Swarm (Olsen et al. 2020). Although the physical mechanisms responsible for most of the field changes take place over time scales longer than the satellite era (the past 20 years), shorter inter-annual fluctuations may be characterised with satellite data alone. 
The Geomagnetic Virtual Observatory (GVo) technique was first developed by Mandea and Olsen (2006) and Olsen and Mandea (2007), as a way of making satellite field measurements easily accessible, through a regression of these into local time series of the field at pre-specified locations. Such GVo time series consist of vector field estimates at mean orbital altitude. Each GVo datum is derived using satellite measurements in the near vicinity of a given GVo location, and within a chosen time window. As for ground-based records, by taking annual differences of these locally derived GVo field estimates, SV time series can be obtained. To address issues of insufficient local time sampling and contamination from external sources such as the magnetospheric ring current and ionospheric current systems (Olsen and Mandea 2007; Beggan et al. 2009; Shore 2013), additional data processing steps, changes to the modelling scheme and de-noising procedures have been incorporated into the GVO algorithm (Hammer et al. 2021a). In order to make robust GVo field estimates, a pre-whitening step is applied wherein main field predictions $\mathbf{B}\left(\mathbf{r}_{\mathrm{GVO}}\right)$ for each data point of position $\mathbf{r}_{\mathrm{GVO}}$ are subtracted off and at a later stage added back for the GVO epoch under consideration. The IGRF model (which has linear SV) is typically used for determining $\mathbf{B}\left(\mathbf{r}_{\mathrm{GVO}}\right)$, however, the choice of model in this pre-whitening step is of little importance. The GVO time series are typically produced at 1-month or 4-month cadence and provided on a global grid of 300 equally spaced locations. Comparisons of the GVo time series with independent ground observatory records and spherical harmonic field models (see Fig. 4) have demonstrated good agreement on all three vector field components (Hammer et al. 2021a). The figure shows SV time series, computed as annual differences, at the three ground observatory stations Ascension Island (East component), Honolulu (Radial component) and Hermanus (South component). Note here that the GVo estimates have been mapped to ground in order to ease the visual comparison.

Figure 5 presents a map of radial SV time series over 1999-2020, at 300 globally distributed GVos, with 4 month cadence. In order to produce such composite time series, the GVos have been mapped to a common altitude of $700 \mathrm{~km}$. This is performed by subtracting from each GVO series the difference between the field at $700 \mathrm{~km}$ and at the mean orbital altitude (separately for each satellite mission), as determined from the CHAOs-7.2 model
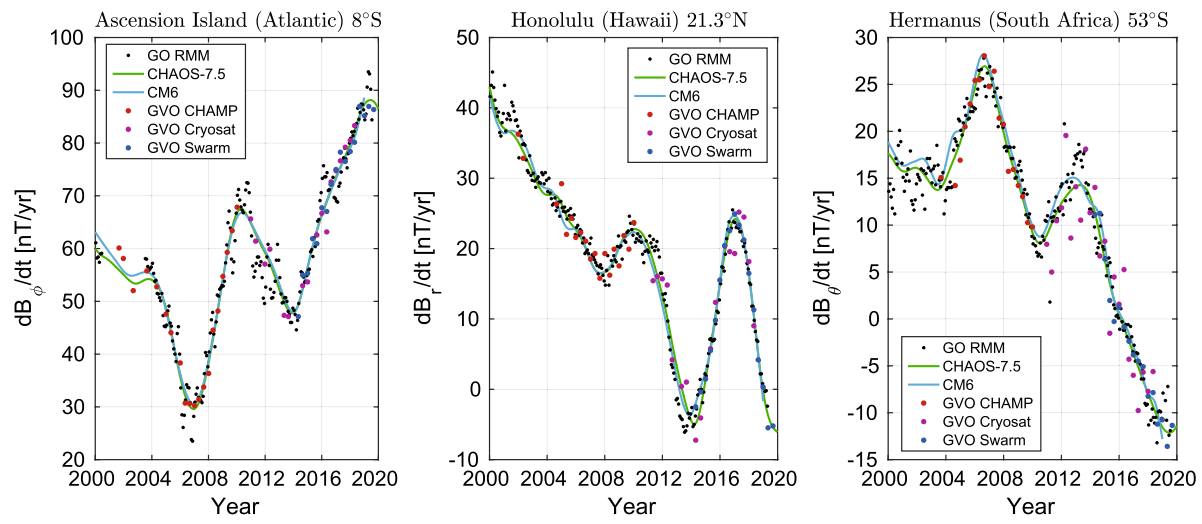

Fig. 4 Comparison of SV times series at three example ground observatories for the East $(\phi)$, Radial $(r)$, and South $(\theta)$ components, respectively. Shown are SV from annual differences of revised monthly means (black dots) (see Olsen et al. 2014), and 4-monthly GVos (mapped to ground) derived from CHAMP (red dots), CryoSat-2 (purple dots) and Swarm (blue dots) measurements. Also shown are predictions of the CM6 (Sabaka et al. 2020) (cyan line) and cHAos-7.5 (Finlay et al. 2020) (green line) models 


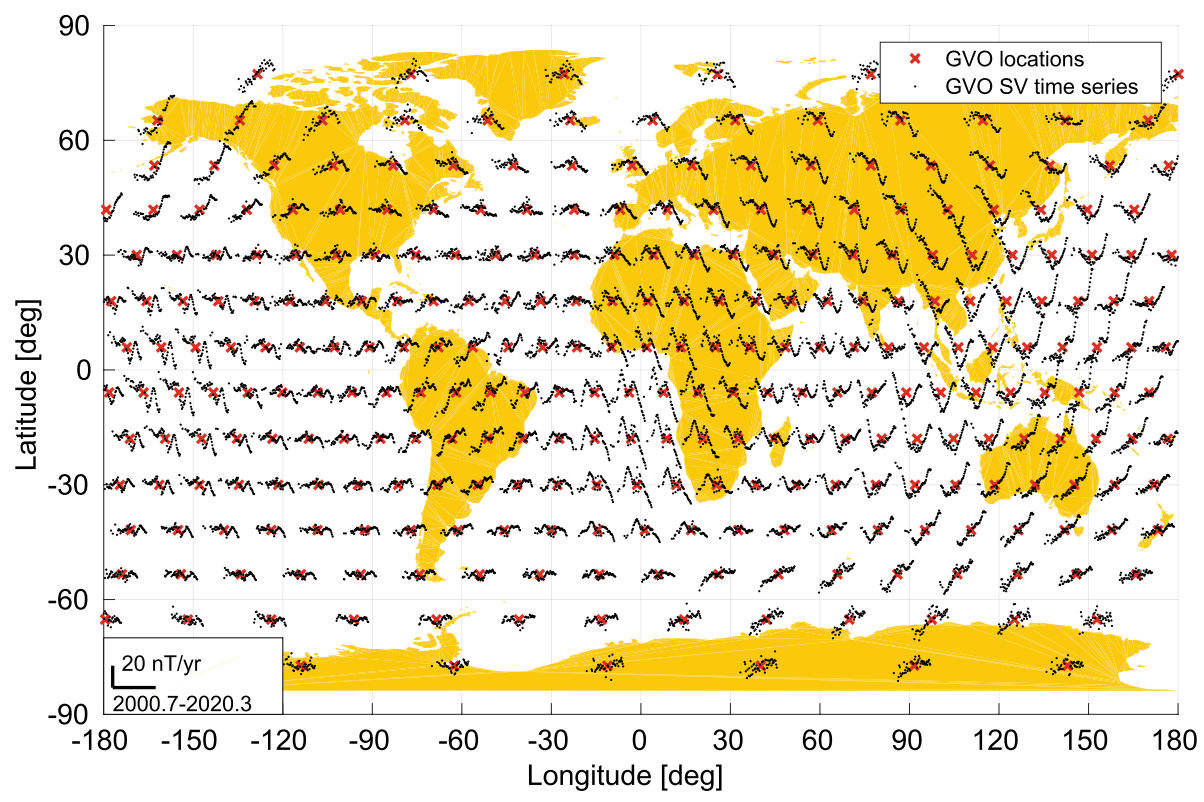

Fig. 5 Composite Gvo time series of the radial SV ( $\partial B_{r} / \partial t$-black dots) over the period 1999-2020, computed as annual differences of 4 monthly GVos mapped at an altitude of $700 \mathrm{~km}$. GVo locations are marked with a red cross. The GVo time series have been derived using measurements from Oersted data (19992004), CHAMP data (2002-2010), CHAOs-6x9 calibrated CryoSat-2 data (2010-2014, see Olsen et al. 2020) and data from the Swarm trio mission (2014-2020). The scale of the time series is shown in the bottom left corner, with the $y$-axis being $20 \mathrm{nT} \mathrm{yr}^{-1}$ and the $x$-axis going from 1999 to 2020. Adapted from Hammer et al. (2021b)

(Finlay et al. 2020). The map in Fig. 5 allows the visual inspection of patterns of subdecadal SV trends and changes. Low latitude regions display particularly strong variations, which do not occur simultaneously over all longitudes. Distinctive " $\Lambda$ " and " $V$ " shaped behaviours occur regionally over time spans ranging from 5 to 10 years. They can be observed for instance over the South-Atlantic region around 2007 and again in 2014, over Indonesia around 2014, and in the Pacific region around 2017. GVos are thus suitable for studying inter-annual and longer field changes at satellite altitude. These likely originate from changes in the outer core fluid motions (see Sect. 4). The GVo dataset, together with their error estimates, is suitable for core flow inversions (Whaler and Beggan 2015; Kloss and Finlay 2019; Rogers et al. 2019) and data assimilation studies that combine information from geodynamo numerical models with signals observed in the GVo time series (Barrois et al. 2018; Huder et al. 2019).

\section{Geomagnetic Field Modelling}

In order to study core field changes occurring over periods from years to decades, the course of action is typically either to analyse magnetic changes as recorded at a particular location, or to analyse spherical harmonic field models (Mandea et al. 2010). 
Although the temporal resolution of ground stations is remarkable, their uneven spatial distribution renders global studies of core field changes challenging (Mandea and Olsen 2006). Alternatively, the construction of field models (e.g., Finlay et al. 2020; Ropp et al. 2020; Sabaka et al. 2020) requires the addition of prior information in order to reduce the non-uniqueness of the geomagnetic inverse problem. Models using a spherical harmonic representation come with attractive mathematical benefits, e.g., orthogonality relations and the addition theorem (Olsen et al. 2010), and have simple means of specifying the model complexity as a function of harmonic degree. However, since spherical harmonics are global support functions, it is preferable to estimate such models with an homogeneous data coverage over the Earth. With real data, specific sources dominate the signals over some regions and it is therefore appealing to consider local methods for studying the magnetic field. We detail in this section local and global methods for modelling the core field.

\subsection{Local Modelling Techniques}

Modelling the geomagnetic field is typically done using spherical harmonic expansions, which remains the preferred tool because of their convenient mathematical properties. However, other modelling strategies focusing on local techniques have been proposed, which have typically been developed for modelling the field on regional scales. Local modelling strategies include (i) considering a limited area with a flat-Earth approximation (Rectangular and Cylindrical Harmonic Analysis, see Alldredge 1981, 1982), or with basis functions defined on a spherical cap (Spherical Cap Harmonic Analysis, see Haines 1985) or (Revised Spherical Cap Harmonic Analysis, see Thébault et al. 2004), (ii) using basis functions such as wavelets (Chambodut et al. 2005; Holschneider et al. 2003), "quasilocal" functions (Lesur 2006), Harmonic Splines (Shure et al. 1982; Geese et al. 2010) or Slepian functions (Beggan et al. 2013; Plattner and Simons 2015), (iii) using spherical harmonic models with localised constraints (Constable et al. 1993), (iv) using equivalent source representations such as dipoles (Mayhew and Estes 1983) and monopoles (Hodder 1982; O’Brien and Parker 1994).

Here, we revisit a local approach called Subtractive Optimally Localised Averages (SOLA). The sola technique allows for localised estimates of the radial field and SV field at the core-mantle boundary (CMB) to be computed as local spatial averages centred on a location of interest (Hammer and Finlay 2019). These local spatial field averages are computed from averaging kernels which are influenced by the used data. The sola technique provides information on the resolution of the field estimate, in the form of the averaging kernels and variance estimates of the locally averaged field. This information is important when trying to resolve smaller length and time scales of the core field signal.

The approach adopted in geomagnetism builds on the method originally developed in helioseismology by Pijpers and Thompson (1994) and is a modified Backus-Gilbert inversion type (Backus and Gilbert 1968, 1970; Pujol 2013). At satellite altitude, the magnetic vector field can be linked to the radial field at the CMB using the Green's functions of the Neumann boundary value problem (Gubbins and Roberts 1983). Focusing on the core field, a dark quiet time selection scheme is typically implemented and field model estimates of the lithospheric field, the magnetospheric and ionospheric fields along with their associated Earth-induced fields are subtracted (Hammer et al. 2021b). Since the CMB radial magnetic field is linearly related to the satellite vector data, a sola estimate at a specified 
target location and time, $\left(\mathbf{r}_{0}, t_{0}\right)$, at the $\mathrm{CMB}$, can be written as a weighted linear combination of the satellite magnetic data (Backus and Gilbert 1970):

$$
\widehat{B}_{r}\left(\mathbf{r}_{0}, t_{0}\right)=\sum_{n}^{N} q_{n}\left(\mathbf{r}_{0}, t_{0}\right) d_{n}\left(\mathbf{r}_{n}, t_{n}\right),
$$

where $q_{n}$ are sola weight coefficients and $d_{n}$ are the satellite data $(n=1, \ldots, N)$. They are related to the radial magnetic field, $B_{r}\left(\mathbf{r}^{\prime}, t_{n}\right)$, integrated over the CMB as (Gubbins and Roberts 1983)

$$
d_{n}\left(\mathbf{r}_{n}, t_{n}\right)=\oint_{S^{\prime}} G\left(\mathbf{r}_{n}, \mathbf{r}^{\prime}\right) B_{r}\left(\mathbf{r}^{\prime}, t_{n}\right) \mathrm{d} S^{\prime},
$$

where the CMB surface element at radius $r^{\prime}$ is $\mathrm{d} S^{\prime}=\sin \theta^{\prime} \mathrm{d} \theta^{\prime} \mathrm{d} \phi^{\prime}$ and the data kernel, $G\left(\mathbf{r}_{n}, \mathbf{r}^{\prime}\right)$, is the spatial derivative of the Green's functions for the exterior Neumann boundary value problem (e.g., Gubbins and Roberts 1983; Barton and Barton 1989). Combining Eqs. (2) and (1), it is possible to write the sola estimate as

$$
\widehat{B}_{r}\left(\mathbf{r}_{0}, t_{0}\right)=\oint_{S^{\prime}} \mathcal{K}\left(\mathbf{r}_{0}, t_{0}, \mathbf{r}^{\prime}\right) B_{r}\left(\mathbf{r}^{\prime}, t_{n}\right) \mathrm{d} S^{\prime},
$$

where $\mathcal{K}\left(\mathbf{r}_{0}, t_{0}, \mathbf{r}^{\prime}\right)$ is a spatial averaging kernel. Thus, the sola weight coefficients together with the data kernel specify the averaging kernel centred on position $\mathbf{r}_{0}$ as

$$
\mathcal{K}\left(\mathbf{r}_{0}, t_{0}, \mathbf{r}^{\prime}\right)=\sum_{n}^{N} q_{n}\left(\mathbf{r}_{0}, t_{0}\right) G\left(\mathbf{r}_{n}, \mathbf{r}^{\prime}\right) .
$$

To determine the sola weight coefficients, an objective function that describes the departure of the spatial averaging kernel towards a pre-specified target kernel is minimised and information from a data error covariance matrix is included (Hammer and Finlay 2019). By varying the target kernel width, sola estimates of different spatial averaging width (i.e. resolution) emerge. Typically, a Fisher distribution is used as a target kernel (Fisher 1953); however, other options are possible (Masters and Gubbins 2003).

When calculating soLA field estimates, data from within a month are commonly used and time-dependence neglected. In order to compute soLA SV estimates, data from within a window (typically 2 years) are used, where the time-dependence is handled using a first order Taylor expansion assumed valid close to a reference time (Hammer et al. 2021b). By taking differences between consecutive sOLA SV field estimates, estimates of the SA at the $\mathrm{CMB}$ can be derived, which is of interest when studying core dynamics (e.g., Finlay et al. 2016; Chi-Durán et al. 2020). In particular, using the high-quality measurements from the Swarm mission, it is possible to take 1-yr differences of sola SV estimates derived from 1 year data windows (Hammer et al. 2021b).

Because the sOLA method allows for local field estimates to be determined, collections of individual solA estimates having the same resolution can be combined into making regional or global maps. Of particular interest for more detailed studies, are regions of strong fluctuations in the field changes observed at low latitudes. In order to examine such changes, the sola method can be used for making SA estimates along the CMB equator in consecutive time steps, such that the evolution of the SA can be mapped. Figure 6 presents time-longitude (TL) plots of the SA evolution on the geographic equator (centred on the Pacific region) at the CMB between 2015.0 and 2020.0 derived from the radial 


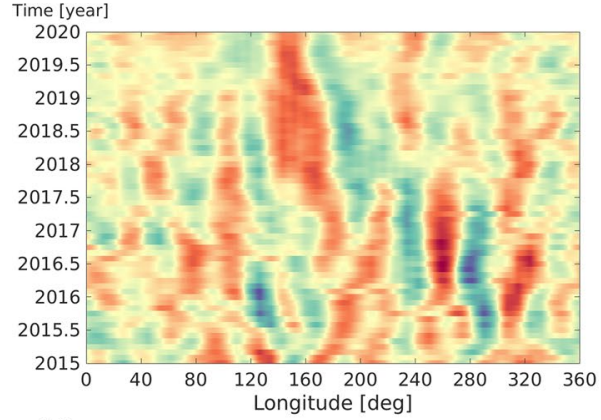

(a) sola radial SA, 1yr time windows, 1 month steps.

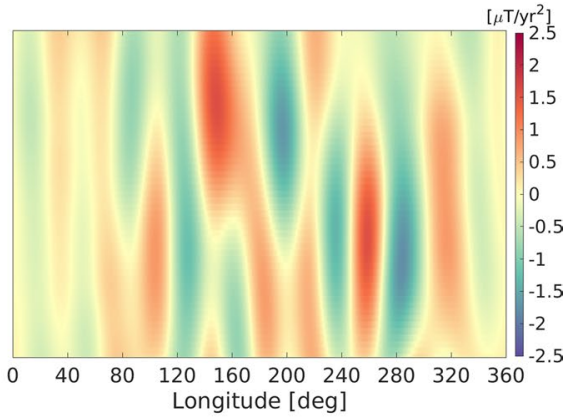

(b) CHAOS-7.2 radial SA.

Fig. 6 Time-longitude plots of the radial SA field along the CMB geographical equator, showing: $1 \mathrm{yr}$ differences of sola SV estimates derived from 1-yr data windows moving in 1 month steps (left), and predictions from the CHAOS-7.2 model for spherical harmonic degrees 1 to 10 (right). Adapted from Hammer et al. (2021b)

field measurements of the Swarm satellites. The left plot shows sola SA estimates derived from 1-yr differences of sola SV determined from 1 year data windows, sliding in 1 month steps, while the right plot shows the SA predictions from the CHAOs-7.2 model for spherical harmonic degrees 1 to $10-$ i.e. a maximum wavelength $\sim 2200 \mathrm{~km}$ at CMB. Here, the solA SA estimates have associated errors estimates of about $0.6 \mathrm{nT} \mathrm{yr}^{-2}$ and averaging kernel widths of $42^{\circ}$, corresponding to length-scales of $2500 \mathrm{~km}$ at the CMB. Comparing the two TL-plots, similar large scale features of the SA evolution with amplitudes of $\pm 2.5 \mathrm{nT} \mathrm{yr}^{-2}$ can be observed in both the SOLA and CHAOS plots. However, the sola plot demonstrates increased temporal resolution and reveals coherent patterns that have been smoothed out by the temporal regularisation of the CHAOS-7.2 model.

In the SOLA TL plot, interesting structures include intense SA patches under Central America, at longitudes from $240^{\circ}$ to $320^{\circ}$, observed going from 2015.5 to 2018 . Also observed are strong side-by-side positive and negative SA features emerging in the Pacific region around 2017 , at longitudes $150^{\circ}$ to $220^{\circ}$, which are seen to have drifted approximately $20^{\circ}$ westwards until 2020. Such rapid variation at low latitudes may be caused by time variations in the liquid outer core flow and could provide important constraints on the equatorial dynamics of the outer core (Kloss and Finlay 2019). Responsible mechanisms for such flows may involve equatorially trapped magnetic, Archimedes and Coriolis (MAC) waves in a stratified layer at the core surface (Buffett and Matsui 2019; Chi-Durán et al. 2020), Magneto-Coriolis modes (Gerick et al. 2020) or equatorial focusing of hydrodynamic waves related to turbulent convection deep within the core (Aubert and Finlay 2019). Possible mechanisms are described in more details in Sect. 4.

\subsection{Global Field Models}

Although building localised representations of the core magnetic field presents numerous advantages, it still relies on a rough separation of the different sources often based on outputs of other's modelling efforts. We therefore present here global magnetic field modelling techniques that simultaneously consider the core and other contributions. Although different types of representation are possible, magnetic field components are most generally described as the negative gradient of potentials of internal or external origins, respectively, $V_{i}$ and $V_{e}$, 


$$
\mathbf{B}(\theta, \phi, r, t)=-\nabla\left\{V_{i}(\theta, \phi, r, t)+V_{e}(\theta, \phi, r, t)\right\},
$$

themselves being decomposed into spherical harmonic as

$$
\begin{aligned}
& V_{i}(\theta, \phi, r, t)=a \sum_{\ell=1}^{L_{i}} \sum_{m=0}^{m=\ell}\left\{g_{\ell}^{m}(t) \cos (m \phi)+h_{\ell}^{m}(t) \sin (m \phi)\right\}\left(\frac{a}{r}\right)^{\ell+1} P_{\ell}^{m}(\cos \theta), \\
& V_{e}(\theta, \phi, r, t)=a \sum_{\ell=1}^{L_{e}} \sum_{m=0}^{m=\ell}\left\{q_{\ell}^{m}(t) \cos (m \phi)+s_{\ell}^{m}(t) \sin (m \phi)\right\}\left(\frac{r}{a}\right)^{\ell} P_{\ell}^{m}(\cos \theta) .
\end{aligned}
$$

Here, $(\theta, \phi, r)$ are colatitude, longitude and radius in geocentric system of coordinates. The $P_{\ell}^{m}$ (.) are Legendre functions. The $g_{\ell}^{m}, h_{\ell}^{m}, q_{\ell}^{m}$ and $s_{\ell}^{m}$ are the Gauss coefficients to be estimated independently for each source, where $h_{\ell}^{0}$ and $s_{\ell}^{0}$ are null. All these Gauss coefficients depend on time $t$. In such a decomposition, it is assumed that there are no sources of magnetic field at the measurement point, which usually is true for ground measurements, but it is not necessarily valid at satellite altitude, in particular in the auroral regions with fieldaligned currents (see Finlay et al. 2017). The internal and external description refers to a sphere of radius $a=6371.2 \mathrm{~km}$, that is an approximation of the Earth's surface. Note that with satellite data, ionospheric fields are generated in between the measurement position and the Earth's surface, introducing some confusion in this simple description. The same difficulty in principle exists for the lithospheric field at the equator, that is clearly outside the reference sphere, but this latter effect is generally neglected.

Over the 20 last years, the nearly continuous series of magnetic satellite missions allow to build particularly high resolution, time-dependent models of the magnetic field. Examples of these models are the Comprehensive models (Sabaka et al. 2016, 2020), CHAOs models (Olsen et al. 2006; Finlay et al. 2020), GRIMm models (Lesur et al. 2008, 2015), for the most known. This list is far from being exhaustive. Special issues associated with the publication of the IGRF generally give a better picture of the diversity and numbers of magnetic models available (see, e.g., Maus et al. 2005b; Finlay et al. 2010b; Thébault et al. 2015; Alken et al. 2020). If these models are different, they agree overall on the core magnetic field and its SV strength and direction at the Earth's surface-see Alken et al. (2020) for more information on statistics of the differences. An example is displayed in Fig. 7, for the epoch 2015.0, of the vertical down components at the CMB from the MCM model (Ropp et al. 2020). It should be noted that, in these images, the spherical harmonic degree is limited to $\ell=13$, and that shorter wavelength signals, that can be dominant and therefore strongly modify the images, are not included.

The agreement between models for the SA remains generally good but they present significant differences in terms of amplitudes and locations of the acceleration patterns. Maps of the vertical down SA component for 2006 and 2018, at the CMB, are displayed in Fig. 8 for two models chaos-7 (Finlay et al. 2020) and мсм (Ropp et al. 2020). As for the SV, the spherical harmonic degree is limited to $\ell=13$, and shorter wavelength signals can significantly modify these maps. Differences between the two models are obvious even if there is a general agreement that strong acceleration patterns are mainly located at the CMB along the equatorial regions, or at all latitudes in between $90^{\circ}$ and $120^{\circ}$ East. Strongest accelerations are often seen under Indonesia or Central America. These acceleration patterns have a footprint at the Earth's surface that is very large, in agreement with those directly observed in Fig. 5.

The observed differences between models result from the way the separation of sources contributing to the measured magnetic field is achieved. The coverage in space 

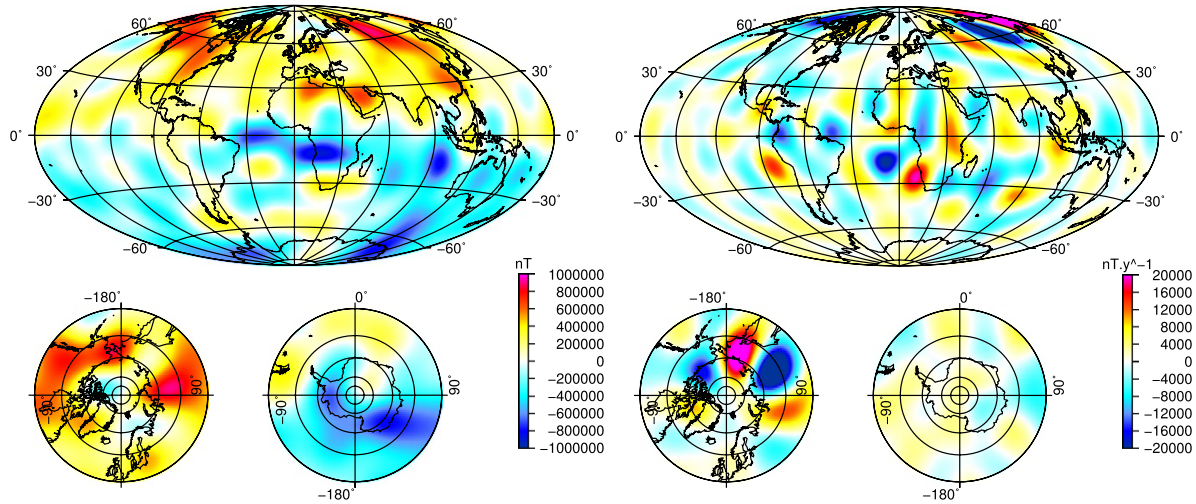

Fig. 7 Vertical down core magnetic field as estimated by the MCM model (Ropp et al. 2020) truncated at spherical harmonic 13 (left) and its secular variation (right). The field has been mapped at the CMB for epoch 2015.0. In both cases, continents are drawn to help location

and time of the combined observatory and satellite data sets is such that for temporal scales of the order of a few days to a few weeks, the separation of large-scale internal fields from external fields is suitable for core field modelling. There may be difficulties for short spatial wavelengths, or short temporal scales but these are generally irrelevant for the core field. One can refer to Lesur et al. (2013) for a description on how fast field variations in time leaks in small wavelength of a nearly static internal field. Satellite data are also contaminated by non-potential magnetic fields [i.e. magnetic field that cannot be described as gradients of potentials as in Eq. (6)]. These signals are mainly due to field aligned electric currents linking the magnetosphere to the ionosphere. These non-potential fields can be co-estimated with other sources (e.g., Sabaka et al. 2016; Lesur et al. 2008), although, even if not modelled, it seems that their contributions do not significantly affect the core field models derived from satellite data. Therefore, the main difficulties that remain for precise core field models are the separation of sources that are seen as internal from the satellite altitude.

From the satellite altitude down, there are firstly the magnetic fields generated in the ionosphere during night-times (day-time data are not usually used for core field modelling). There have been several attempts to model these fields, particularly at high latitudes (e.g., Lesur et al. 2008), but they remain difficult to handle because satellites generally fly at nearly sun-synchronous orbits and therefore over a few days acquire data only on a narrow local time window. It should be noted that because the Earth rotates under the ionosphere that is locked in local time, the ionospheric signals may preferentially affect zonal Gauss coefficients (i.e. $g_{l}^{m}$ and $h_{l}^{m}$ with $m=0$ ) of the core contribution. Secondly, there are the fields generated in the lithosphere. So far, there has been no successful attempt to separate the lithospheric field from the core field. It follows that the contribution of the lithospheric field is ignored at long wavelengths. At shorter wavelengths, from spherical harmonic degree higher than $\ell=16$, where the lithospheric field is dominant at the Earth's surface, the static core field contribution is unknown. At the same altitude, signals generated in oceans can be well estimated when linked to tides because of their well established periodicities (Grayver and Olsen 2019). However, the signals associated with general oceanic circulation have not been isolated yet. There are probably other sources in the crust that have not been identified, such as possibly the 

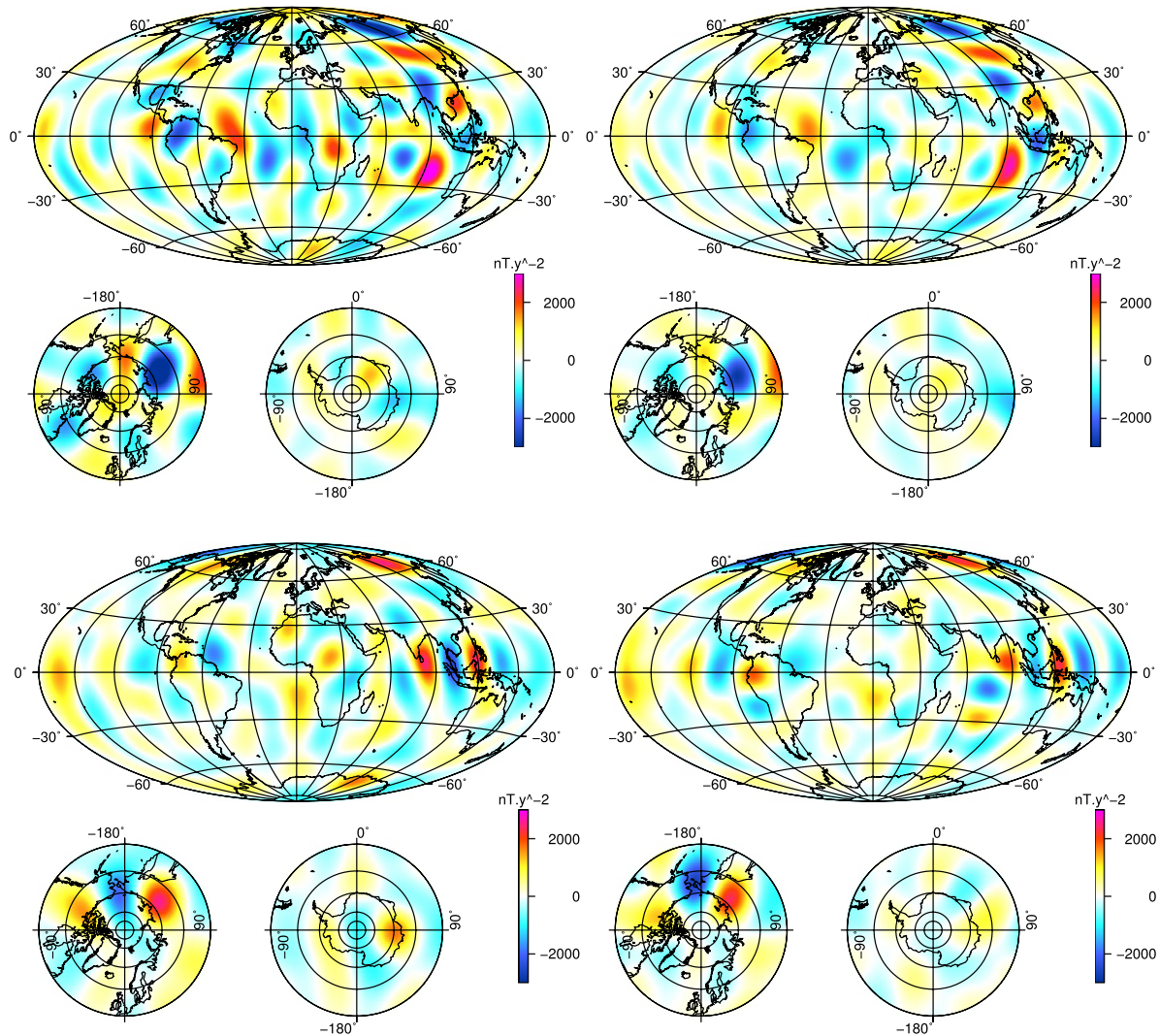

Fig. 8 Vertical down core magnetic field acceleration as estimated by the MCM model (Ropp et al. 2020) (left) or chaOs-7 model (Finlay et al. 2020) (right), truncated at the spherical harmonic $\ell=13$. The field has been mapped at the CMB for year 2006 (first row) and 2018 (second row). Continents are drawn to help location

water cycle that has a strong signature in gravity data. However, the field induced in the conductive crust and mantle by the time varying external fields remains the major difficulty that precludes the derivation of accurate core field models as both contributions overlap over a large range of spatial and temporal scales (see, e.g., Olsen et al. 2005a). It is presently the isolation of long periods external fields (and their induced counterparts) that sets the temporal resolution of core field models. Ambiguities may remain at the decadal period of the solar cycle (and its harmonics), and more importantly, we lack accurate understanding of the strong semi-annual and annual periodicities that arise naturally in ionospheric and external fields.

Generally, the strategy used to separate these various "internal" fields from the core field is based on a careful data selection, a partial modelling of the different contributions, and often, a constraint (i.e. a damping or regularisation) set on the core field contribution such that it does not vary too rapidly in time. This approach has been used for a long time, and examples of application to satellite data can be found in, e.g., Olsen et al. (2006) and Lesur et al. (2008) or more recently Sabaka et al. (2020) and Finlay et al. (2020). The characteristic of this approach is that the core magnetic field SV and SA 
are averaged over time. Furthermore, their temporal resolutions vary depending on the spatial scale. As shown in Fig. 9, with these approaches, the acceleration is likely underestimated at short periods and small wavelengths-i.e. from spherical harmonic degree $\ell=5$ up. An alternative approach has emerged, pioneered by McLeod (1996) and Gillet et al. (2013), where the temporal constraints applied to construct the core field model is derived from what is known of the spatial and temporal behaviour of each source contributing to the observed magnetic field. The temporal constraints are imposed either through a priori time cross-covariances (Huder et al. 2020), or by time-stepping associated stochastic equations (Huder et al. 2019; Baerenzung et al. 2020; Ropp et al. 2020). As a result, the modelled SV and SA are not averaged over time by construction, to the cost of having models with large posterior uncertainties (Gillet et al. 2013; Holschneider et al. 2016). We point for instance to Ropp et al. (2020) where outputs from numerical dynamos are used as prior spatial information on the core field behaviour. Whatever modelling technique is used, some temporal averaging may still exist in the models due to the imperfect data coverage. In particular, the nearly continuous satellite era extends only over the last 20 years precluding robust estimation of temporal periodicities in the core exceeding 10 years.

All these modelling techniques rely strongly on what is assumed by the modeller regarding the magnetic field generated by different sources, and in particular by the Earth's core. To better understand the dynamics of the liquid outer core, it is therefore important to define how fast the core field varies with time at the Earth's surface. The fastest variations in the core field are necessarily smoothed out by (i) magnetic diffusion within the fluid core and (ii) induction in the conductive mantle. However, the associated cut-off periods are not well known. On the core side, the most extreme numerical simulations of the geodynamo suggest that it could be of the order of 1 year. This is hard to confront immediately to magnetic data because of the dominant external signals towards high frequencies (see Fig. 3). On the mantle side, the cut-off period remains difficult to estimate because of the poorly constrained lowermost mantle conductivity (e.g., Kuvshinov et al. 2021). This cut-off value is likely less than 1 year (see Jault 2015). It is interesting to see how much variability has been accepted for the core field or its SV in the currently available models. Both quantities are characterised by the SV and SA time scales, as defined by, respectively,

Fig. 9 Intensity of the SA at the core surface $\left(\log _{10}\right.$ of $\left.\mathrm{nT} \mathrm{yr}^{-2}\right)$ as a function of the period and the spherical harmonic degree, for the cHAOs-7 field model (Finlay et al. 2020). Adapted from Gillet (2019)

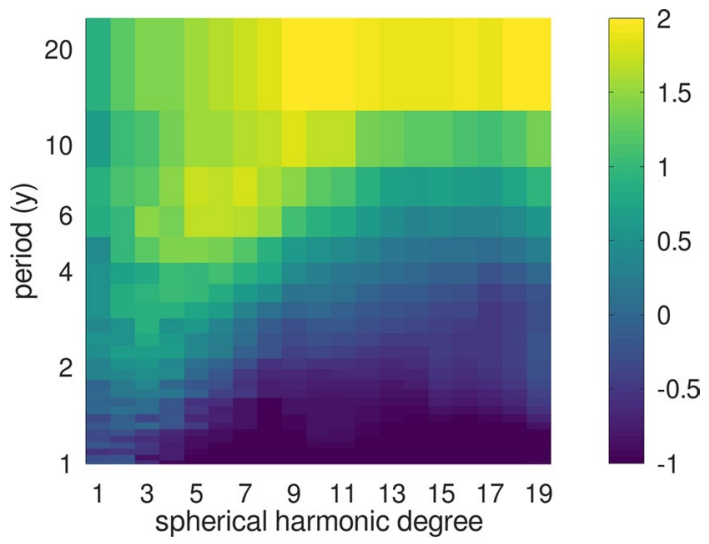




$$
\tau_{\mathrm{sv}}(\ell)=\sqrt{\frac{\sum_{m}\left(g_{\ell}^{m}\right)^{2}+\left(h_{\ell}^{m}\right)^{2}}{\sum_{m}\left(\dot{g}_{\ell}^{m}\right)^{2}+\left(\dot{h}_{\ell}^{m}\right)^{2}}}
$$

and

$$
\tau_{\mathrm{sa}}(\ell)=\sqrt{\frac{\sum_{m}\left(\dot{g}_{\ell}^{m}\right)^{2}+\left(\dot{h}_{\ell}^{m}\right)^{2}}{\sum_{m}\left(\ddot{g}_{\ell}^{m}\right)^{2}+\left(\ddot{h}_{\ell}^{m}\right)^{2}}} .
$$

In these equations, we use the notations $\dot{x}=\partial x / \partial t$ and $\ddot{x}=\partial^{2} x / \partial t^{2}$. From the combined analysis of magnetic field models and numerical simulations, Christensen et al. (2012) propose, for $1<\ell \leq 13, \tau_{\mathrm{sv}}(\ell) \simeq 480 / \ell$ (in fair agreement with Lhuillier et al. 2011) and $\tau_{\mathrm{sa}}(\ell) \simeq 11$ years for $\ell \leq 10$. With the new generation of numerical geodynamo simulations (Aubert 2018), the $\tau_{\mathrm{sa}}$ value decreases down to sub-decadal periods (for the largest length-scales) as conditions closer to Earth-like are reached (Aubert and Gillet 2021).

Because the SV energy is relatively well constrained by observations, $\tau_{\mathrm{sa}}$ depends directly on the SA energy. The latter can be decomposed, from the induction equation in the fluid outer core

$$
\dot{\mathbf{B}}=\nabla \times(\mathbf{u} \times \mathbf{B})+\eta \Delta \mathbf{B},
$$

into three terms as

$$
\ddot{\mathbf{B}}=\nabla \times(\dot{\mathbf{u}} \times \mathbf{B})+\nabla \times(\mathbf{u} \times \dot{\mathbf{B}})+\eta \Delta \dot{\mathbf{B}} .
$$

Here, $\mathbf{u}$ is the flow in the Earth's liquid outer core, and $\eta$ is the magnetic diffusivity. The first term in the right-hand side of Eq. (9) is the SV generated by the advection of the magnetic field by the flow, whereas the second term is linked to magnetic diffusion. In Eq. (10), the first term in the right-hand side is the SA generated by the flow acceleration, the second is the SA generated by the flow advecting the SV, and the last term is the contribution from diffusion. Results from numerical dynamo experiments suggest that the two first terms in Eq. (10) strongly dominate the last one, and that the first term most often dominates over the second (Aubert 2018; Aubert and Gillet 2021). The second term of the same decomposition, neglecting diffusion, was used by Lesur et al. (2010) to estimate a lower bound for the acceleration energy. Presented in Fig. 10, it can be seen as an upper limit for an acceptable acceleration time scale $\tau_{\mathrm{sa}}(\ell)$. All recent magnetic field models have acceleration time scales for spherical harmonic degree up to $\ell=13$ well below this limit.

In the same figure is shown $\tau_{\mathrm{sa}}(\ell)$ derived from the Coupled-Earth numerical dynamo model (Aubert et al. 2013). For this dynamo simulation (as for the ones used by Christensen et al. 2012), transient phenomena such as SA pulses are excluded because of a too weak magnetic to kinetic energy ratio. This tends to produce larger estimates of $\tau_{\mathrm{sa}}$ (see also Sect. 4 for possible physical interpretations of SA pulses). Therefore, except for spherical harmonic degree $\ell=1$ (where the Coupled-Earth dynamo model is known to present too weak SV), it is expected that the estimates for $\tau_{\mathrm{sa}}$ from field models remain below the one derived from the Coupled-Earth model. As seen in Fig. 10, this weak constraint is respected by recent magnetic field models (as shown for the CHAOs-7 and MCM models). For the CHAOs series of models, the progress in modelling and the precise adjustment of the smoothing constraints led to a significant decrease of the time scales towards large spherical harmonic degrees. The MCM model that uses alternative temporal representation (see above) presents even lower time scale for degrees $\ell$ from 6 to 13, but slightly larger values 


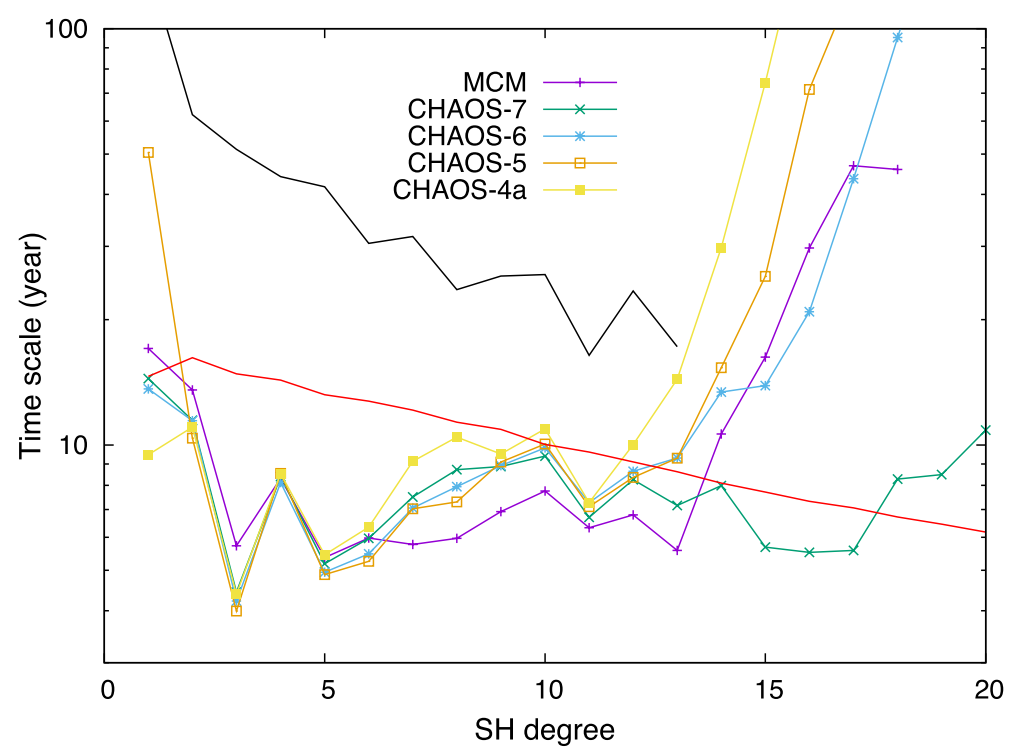

Fig. 10 Acceleration time scales $\tau_{\mathrm{sa}}(\ell)$ as a function of spherical harmonic degree $\ell$, for year 2006, and as estimated with successive versions of chaOs field model (Finlay et al. 2020) and the mCM model (Ropp et al. 2020). Are also shown a characteristic time scale derived from the Coupled Earth numerical dynamo model (red line-Aubert et al. 2013), and a time scale obtained neglecting the flow acceleration in the core and the diffusion (black line-see the main text)

at the lowermost degrees. It is reassuring that all models seem to agree for intermediate length-scales ( $\ell$ around 5). Clearly, above degree $\ell=13$ estimates for $\tau_{\mathrm{sa}}$ are poor for all models (the SA power drops as it is badly constrained). However, there is no insurance yet that at lower spherical harmonic degrees, the time scales estimates are exact. It is possible that some fast variations of the core field are accounted for within the induction part of the models, or that rapid induced and ionospheric signals are modelled as generated in the core. We note, however, that the typical 11-yrs value for the acceleration time scale obtained in Christensen et al. (2012) seems overestimated, in agreement with the latest simulations of the geodynamo.

These time scales are varying with time over the satellite era. Figure 11 presents temporal evolution of $\tau_{\mathrm{sa}}(\ell)$ derived from the MCM model (Ropp et al. 2020) and the CHAOS-7 model (Finlay et al. 2020) from 2000 to 2020. Edge effects are visible for the MCM model in 2000 and 2020. Both models show a significant variability up to spherical harmonic degree $\ell=6$, above which the CHAOS-7 SA appears smoother. However, values obtained with MCM are associated with large uncertainties. A good agreement in terms of variability is found for degrees 3, 4 and 5. Discrepancies are important for the spherical harmonic degree $\ell=1$ where part of the variability coincides with satellite data availability. Much more variability is obtained with the CHAOS-7 model, and this difference with the MCM model is probably due to different way of handling high latitude night-time ionospheric signal and/or induced fields.

As described above, the variability of the magnetic field is a key information to better understand the outer core dynamics. Progresses have been tremendous in terms of spatial and temporal description of this variability. In particular, this leads to the possibility to identify waves and other transient phenomena in the core. 

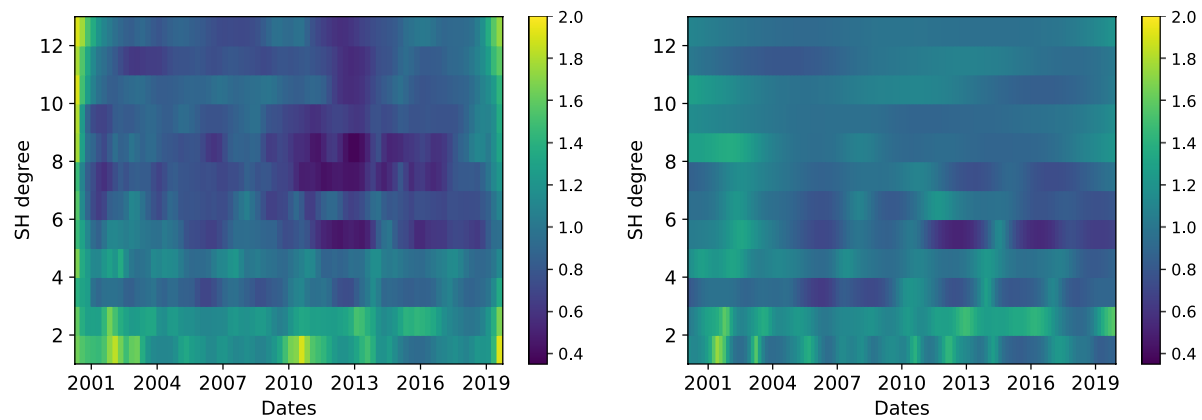

Fig. 11 Time scales from 2000 to 2020 , for a spherical harmonic degree range $\ell \in[1 ; 13]$ (units in $\log _{10}$ of years). Time scales are shown for the MCM model (left) and cHAOs-7 model (right)

\section{Possible Sources of Inter-annual Geomagnetic Changes}

The apparent periodicity of SA pulses (Chulliat and Maus 2014; Finlay et al. 2016) has motivated the search for waves as an origin to rapid SV changes. In the presence of global rotation, the fluid response to transient perturbations consists of inertial waves, whereas, within an ambient magnetic field, the responses to perturbations are made of Alfvén waves. For the former waves, the restoring force comes from the Coriolis force, while for the latter, it is the Lorentz force (for a review, see Finlay et al. 2010a; Jault and Finlay 2015). Their typical periods are comparable with the rotation and Alfvén times, respectively:

$$
\tau_{\Omega}=1 / 2 \Omega \text { and } \tau_{A}=c / V_{A},
$$

with $\Omega \simeq 7.3 \times 10^{-5} \mathrm{rad} \mathrm{s}^{-1}$ the Earth's rotation rate, $c=3485 \mathrm{~km}$ the core radius, and $V_{A}=B / \sqrt{\rho \mu}$ the Alfvén speed $\left(\rho \simeq 10^{4} \mathrm{~kg} \mathrm{~m}^{-3}\right.$ is the core density and $\mu=4 \pi \times 10^{-7}$ $\mathrm{H} \mathrm{m}^{-1}$ the magnetic permeability). For a magnetic field intensity $\approx 5 \mathrm{mT}$ within the core (Gillet et al. 2010), one gets $\tau_{A} \approx 2$ years that is much longer than $\tau_{\Omega}$, as measured by the Lehnert number Le $=\tau_{\Omega} / \tau_{A} \approx 10^{-4}$. For planetary scale Alfvén waves to propagate, their periods must be much less than the diffusion time $\tau_{\eta}=c^{2} / \eta$ (with $\eta \simeq 1 \mathrm{~m}^{2} \mathrm{~s}^{-1}$ the magnetic diffusivity). This is the case in the Earth's core, as measured by the Lundquist number $\mathrm{Lu}=\tau_{\eta} / \tau_{A} \approx 10^{5}$.

When both rotation and magnetic field are present, the response to a planetary-scale perturbation by inertial waves is thus much faster than that by Alfvén waves. The predominance of the Coriolis force in the momentum budget has two important consequences. First, transient flows tend to be almost invariant along the rotation axis (Jault 2008), or quasi-geostrophic (QG). Second, for Le $\ll 1$ there should exist no large length-scales QG Alfvén modes in Earth's core, but instead magneto-Coriolis (MC) modes (Hide 1966). These eigen-modes in the presence of global rotation and an imposed field divide for Le $\ll 1$ into slow and fast modes, whose periods are commensurate with, respectively,

$$
\tau_{\mathrm{MC}}^{s} \approx \tau_{A}^{2}(L / c)^{2} / \tau_{\Omega} \text { and } \tau_{\mathrm{MC}}^{f} \approx \tau_{\Omega} c / L
$$

with $L$ a typical length-scale (for a review, see Finlay et al. 2010a). Fast QG-MC modes are only weakly sensitive to the magnetic field and correspond to Rossby modes (i.e. axially invariant inertial modes, see Zhang et al. 2001). At large length-scales, their periods remain much smaller than $\tau_{A}$ (it spans approximately the range between 0.1 and 2 months 
for azimuthal wave numbers $m \leq 10$ and radial wave numbers $N \leq 5$ ). Because their ratio of magnetic to kinetic energy is weak, their detection in magnetic data is very difficult (furthermore, in the monthly period range, the core signal is hindered by external sourcessee Fig. 3). Conversely, slow QG-MC (or 'magnetostrophic') modes are dominated by the magnetic energy, and their period reaches centuries to millennia. Thus, a priori, they cannot explain SA pulses (but see below).

Nevertheless, one particular family of Alfvén modes exists. These 'torsional' modes are organised as cylinders co-axial with the rotation axis (geostrophic cylinders). For these modes, the projection of the Coriolis force vanishes, so that the fluid response to a perturbation naturally comes from the Lorentz force (Braginsky 1970). Detected from geomagnetic field models at a period of 6-yrs (Gillet et al. 2010), their outward propagation across the fluid core in about 4 years constrains the intensity of the field in the bulk of the core. However, their flow perturbation magnitude (of r.m.s. $\approx 0.3-0.5$

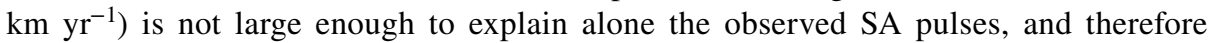
non-axisymmetric flow contributions about 3 to 5 times larger are required (Gillet et al. 2015, 2019; Kloss and Finlay 2019).

Several possibilities have been proposed to explain such intense non-zonal motions at inter-annual periods, among which are waves involving a stratified layer at the top of the core. The restoring force then comes from an interplay between the magnetic, Archimedes and Coriolis forces. Such 'MAC' waves are appealing in the context of a heat flux at the CMB weaker than the adiabatic flux, a scenario made possible with a relatively high thermal conductivity of the core (Pozzo et al. 2012; Ohta et al. 2017) and motivated by seismological studies (Helffrich and Kaneshima 2010). The role of buoyancy in the stable layer is governed by the steepness of the density profile $\rho^{\prime}(r)=\rho(r)-\rho_{0}(r)$ below the CMB (with $\rho_{0}$ the reference density profile for stability, the adiabatic one in the Boussinesq approximation). It is measured by the Brunt-Vaïsala frequency

$$
N=\sqrt{-\frac{g}{\rho} \frac{\mathrm{d} \rho^{\prime}}{\mathrm{d} r}},
$$

where $g(r)$ is the gravity acceleration. For reduced models of a hidden ocean at the top of the core, the control parameters are only the density jump $\Delta \rho$ across the layer, and the height $H$ of the stratified layer (Braginsky 1993, 1999). By tuning $N$ (or $H$ and $\Delta \rho$ ), it is possible to match the inter-annual time scales of interest for SA pulses (e.g., Chulliat et al. 2015; Chi-Durán et al. 2020). Note that, the configuration of the background field has a significant influence on the spatial distribution of the waves (see Knezek and Buffett 2018). In particular under some conditions, MAC waves may be trapped in the equatorial belt (Buffett and Matsui 2019), as is the majority of SA pulses witnessed over the satellite era.

Meanwhile, the existence of a stratified layer is still a debated issue from several aspects: seismology (Irving et al. 2018), geodynamo simulations (Gastine et al. 2020) and high-pressure-temperature experiments (Konôpkovà et al. 2016). Furthermore, rapid SV events have been found in geodynamo simulations, in the absence of any stratification, as the regime of parameters gets closer to Earth's conditions (Aubert 2018). There, they are interpreted as the signature of QG Alfvén waves carried by intense magnetic field lines (Aubert and Finlay 2019). Such Alfvén waves can exist in spite of the Coriolis force because of their reduced extension in the cylindrical radial direction. If the Coriolis force determines their QG nature, it seems to only marginally affect their period, slightly above $\tau_{A}$ (Aubert and Gillet 2021). Nevertheless, the complexity of the background field within the dynamo simulations, that present strong heterogeneities, 
renders more subtle to decipher the roles played by the Coriolis, Lorentz and diffusion terms along the wave propagation (Aubert 2019). Even though SV field models are limited in spatial resolution, QG Alfvén waves might be detectable as they reach the equatorial area, because of the spherical geometry of the core that (i) focuses the wave energy and (ii) projects sharp gradients in the cylindrical radial axis onto smoother spatial variations at the spherical boundary. The detection of such QG Alfvén waves in dynamo simulations is supported by the finding of Gerick et al. (2020): the periods of the slow QG-MC waves decrease to inter-annual values as the spatial complexity of its modes increases. These eigen-solutions furthermore focus the SV signals in the equatorial belt and may explain the minimum of radial velocity at $\pm 10^{\circ}$ in latitude recovered from magnetic data (Gillet et al. 2015). They may therefore be the elementary components needed to understand the SA derived from satellite data. The several families of waves discussed above, and their main characteristics regarding SA events, are summarised in Table 1.

One should keep in mind some caveats when interpreting the strong SA components in models of the core magnetic field. First, a significant part of the magnetic signal is likely associated with unresolved patterns, consequence of the advection process (Eqs. 9, 10) that nonlinearly mixes wavelengths (e.g., Pais and Jault 2008; Gillet et al. 2019). This reduces the resolution of the reconstructed core dynamics. Furthermore, the quasi-periodic nature of observed SA pulses (Soloviev et al. 2017) remains questionable, because of the limited spatio-temporal resolution of geomagnetic field models (Gillet 2019), inherent in the difficult separation of magnetic sources (see Sect. 3.2 and also Finlay et al. 2017). Indeed, when looking at rapid SV changes from magnetic field models, the most intense patterns necessarily show up at intermediate length-scales, as towards short wavelengths fast variations are generally smoothed out (see Fig. 9 and Sect. 3.2).

Finally, whatever the source of SA pulses, the core dynamics do generate a magnetic field with a temporal spectrum behaving approximately as $S(f) \propto f^{-4}$ at observatory sites, or equivalently (in the case of an insulating mantle) through series of geomagnetic Gauss coefficients at the core surface. This spectral property has been recovered in dynamo simulations, even when run at parameters far from Earth-like values, for the dipole moment (Olson et al. 2012; Buffett and Matsui 2015), as well as for non-dipole coefficients (Bouligand et al. 2016). However, the range of frequencies relevant to interpret inter-annual changes must also be considered in comparison with the Alfvén time $\tau_{A}$ on the one side, and with the turn-over time $\tau_{U}=c / U$ on the other side. Here, $U$ is the typical flow speed in the core, and $c$ the core radius, leading for $U \approx 20 \mathrm{~km} . \mathrm{yr}^{-1}$ to $\tau_{U} \approx 150 \mathrm{yr}$. The spectral range in $f^{-4}$ for the Earth's core is found for frequencies in the range $\tau_{A} \leq 1 / f<\tau_{U}$. It is numerically very expensive to reach geophysical values of the Alfvén number $A l=\tau_{A} / \tau_{U} \approx 10^{-2}$ in numerical geodynamo simulations (Schaeffer et al. 2017). When $A l=O(1)$, all the processes at work in Earth's core at periods from a few years to a century are indistinguishable. It is only recently, by using specific parameterisation of turbulent processes, that values of $A l$ significantly lower than $O(1)$ have been achieved (Aubert et al. 2017). Only such extreme numerical experiments present a dynamics usable to replicate the physics of rapid SV changes. They show that torsional waves propagate predominantly outward, as recovered from magnetic records and are constantly excited. Furthermore, as numerical geodynamo parameters get closer to Earth's conditions, jerk events become ubiquitous, and the range where the temporal spectrum for the SA is approximately flat (or equivalently that of the main field $\propto f^{-4}$ ) becomes wider (Aubert 2018; Aubert and Gillet 2021). 


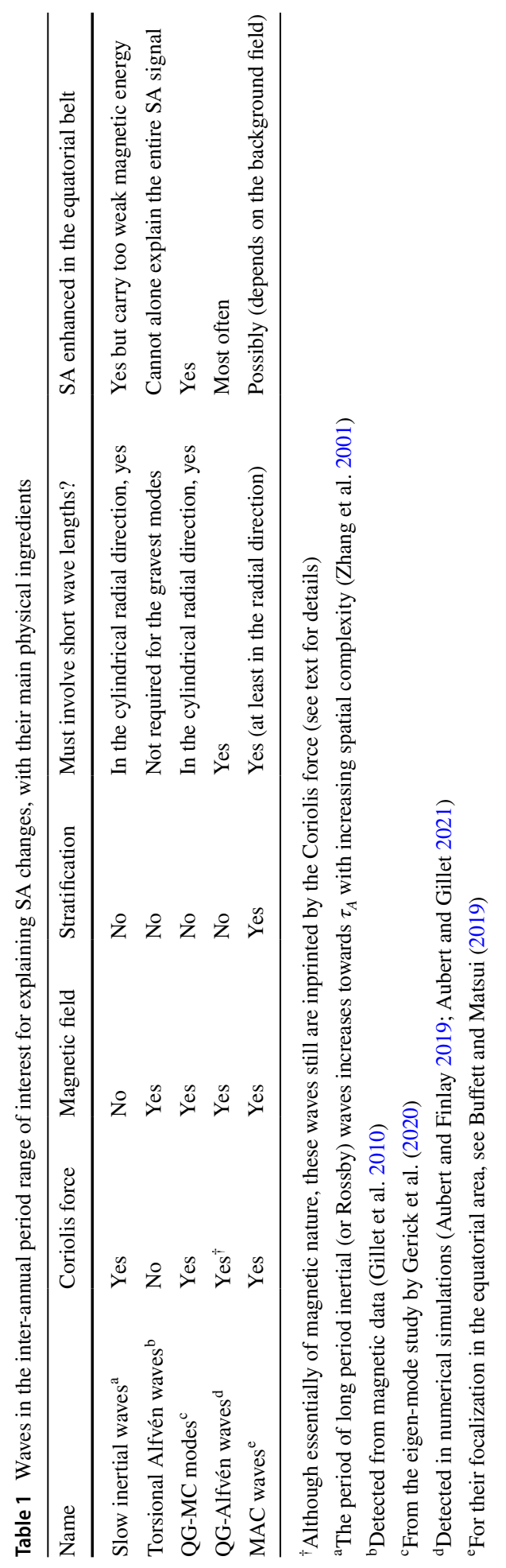




\section{Conclusions}

In this short overview, we have described first the observational evidence of fast variations of the magnetic field generated in the liquid outer core of the Earth. These results directly derive from processing steps applied to magnetic observatory or satellite data. Both show episodes of fast variations in the geomagnetic field secular variation, mainly at mid- and low-latitudes. The application of modelling techniques either localised or global shows that these fast variations are mainly due to strong, localised, spots of acceleration at the core mantle boundary. These are generally, but not always, located close to the equator under Indonesia or Central America, or in the northern hemisphere along the meridian $100^{\circ} \mathrm{E}$.

In order to understand these observations, one of the pressing questions that must be addressed is the temporal variability acceptable for core field models. An answer to this question will necessarily come from a combination of theoretical approaches that provide the possible characteristics (periodicities, strength, location) of a signal generated in the core, and an observational approach that extracts core field signatures from the complex mix of signals observed at the Earth's surface or in its immediate environment. Regarding this latter approach, the data coverage provided by satellite missions is such that the main difficulty to answer this question is the characterisation and separation of the core field from sources of internal origins, as seen from satellite altitudes. The fields induced in the mantle by rapidly varying external fields and ionospheric contributions are the sources, internal to the satellite sampling region, most difficult to separate.

Current field models present time scales for the secular acceleration much shorter than previously thought, of the order 10 to 15 years for the largest wavelengths (spherical harmonic degrees 1 and 2), down to 6 to 7 years from spherical harmonic degrees 5 or 6 up. They present also a temporal variability that has not been yet studied, but certainly carries information on the core dynamics. However, these time scale values may still be overestimated and hide part of the complexity of the underlying dynamics. In particular, $\tau_{\mathrm{sa}}$ remains much larger than the expected cut-off period (1 year or less) where the magnetic core field variations would be smoothed out by the magnetic diffusion within the core or by electrical currents in the conductive mantle. Nevertheless, it is possible that the obtained $\tau_{\mathrm{sa}}$ values are geophysically relevant since extrapolation of the current dynamo runs the closest to Earth's conditions suggest that time scales of $\approx 7$ years are possible.

However, these dynamo simulations do not explore configurations where there is a stratification at the top of the core and alternative processes for generating inter-annual motions. We have tried here to give an overview of the possible transient responses that have been documented so far. It is not an exhaustive picture, as we ignore possible forcings from Earth nutation and precession that may be of importance as the Earth's core deviates from a perfect sphere (Le Bars et al. 2015). In a turbulent regime, these forcings may also lead to QG flows and trigger dynamics at inter-annual time scales.

Currently, the scientific community has a limited knowledge on the three-dimensional distribution of the mantle conductivity. In addition, we lack data at Earth's surface to better describe ionospheric fields, and we have difficulties to separate lithospheric or oceanic contributions from the core field. It follows that the most promising path to better describe the core field dynamics is to identify through theoretical studies their expected main characteristics (e.g., propagating waves, time scales) and try to identify their signals in observatory and satellite data sets. This can be done through a co-estimation with core surface flow models (e.g., Barrois et al. 2018; Baerenzung et al. 2018), or more sophisticated assimilation approaches based either on dynamo simulations (e.g., Sanchez et al. 2019) or on 
reduced QG equations (see Jault and Finlay 2015; Gerick et al. 2020; Jackson and Maffei 2020), although these have not led yet to any application to geophysical data. Given the time scales involved in the core field processes and the core complex spatio-temporal dynamics, it is crucial to continuously measure the magnetic field with satellites carrying magnetometers and in magnetic observatories. Some information content on the core dynamics might be obtained from other types of data, such as Earth's rotation, gravity or geodesy. The core variations' signature in these data is certainly very weak; however, their use in synergy with magnetic data will surely improve our understanding of the liquid core dynamics.

Acknowledgements We thank the anonymous reviewer for remarks that helped us to improve the manuscript. This work was partially supported by the CNES. It is based on observations with magnetometers aboard the Swarm mission. The contribution of NG was also funded by ESA in the framework of EO Science for Society, through contract 4000127193/19/NL/IA (SWARM + 4D Deep Earth: Core). For MM the research leading to these results has received funding from the European Research Council (ERC) GRACEFUL Synergy Grant No. 855677. MDH was supported by the European Research Council (ERC) under the European Union's Horizon 2020 research and innovation programme (Grant Agreement No. 772561) and also partly by Swarm DISC activities, funded by ESA Contract No. 4000109587. This is the IPGP contribution number 4243 .

Open Access This article is licensed under a Creative Commons Attribution 4.0 International License, which permits use, sharing, adaptation, distribution and reproduction in any medium or format, as long as you give appropriate credit to the original author(s) and the source, provide a link to the Creative Commons licence, and indicate if changes were made. The images or other third party material in this article are included in the article's Creative Commons licence, unless indicated otherwise in a credit line to the material. If material is not included in the article's Creative Commons licence and your intended use is not permitted by statutory regulation or exceeds the permitted use, you will need to obtain permission directly from the copyright holder. To view a copy of this licence, visit http://creativecommons.org/licenses/by/4.0/.

\section{References}

Abarca del Rio R, Gambis D, Salstein D (2000) Interannual signals in length of day and atmospheric angular momentum. Ann Geophys 18:347-364

Alexandrescu M, Courtillot V, Le Mouël JL (1996) Geomagnetic field direction in Paris since the midsixteenth century. Phys Earth Planet Inter 98:321-360

Alexandrescu M, Courtillot V, Le Mouël JL (1997) High-resolution secular variation of the geomagnetic field in western Europe over the last 4 centuries: comparison and integration of historical data from Paris and London. J Geophys Res 102:20245-20258

Alken P, Thebault E, Beggan C, Aubert J, Baerenzung J, Brown WJ, Califf S, Chulliat A, Cox G, Finlay CC et al (2020) Evaluation of candidate models for the 13th generation international geomagnetic reference field. Earth Planets Space 73:1-21

Alldredge L (1981) Rectangular harmonic analysis applied to the geomagnetic field. J Geophys Res Solid Earth 86(B4):3021-3026

Alldredge LR (1982) Geomagnetic local and regional harmonic analyses. J Geophys Res Solid Earth 87(B3):1921-1926

Alldredge LR (1984) A discussion of impulses and jerks in the geomagnetic field. J Geophys Res $89: 4403-4412$

Aubert J (2014) Earth's core internal dynamics 1840-2010 imaged by inverse geodynamo modelling. Geophys J Int 197:1321-1334

Aubert J (2018) Geomagnetic acceleration and rapid hydromagnetic wave dynamics in advanced numerical simulations of the geodynamo. Geophys J Int 214:531-547. https://doi.org/10.1093/gji/ggy161

Aubert J (2019) Approaching Earth's core conditions in high-resolution geodynamo simulations. Geophys J Int 219(Supplement_1):S137-S151

Aubert J, Finlay CC (2019) Geomagnetic jerks and rapid hydromagnetic waves focusing at Earth's core surface. Nat. Geosci 12(5):393-398 
Aubert J, Gillet N (2021) The interplay of fast waves and slow convection in geodynamo simulations nearing earth's core conditions. Geophys J Int 225(3):1854-1873

Aubert J, Finlay CC, Fournier A (2013) Bottom-up control of geomagnetic secular variation by the Earth's inner core. Nature 502(7470):219-223

Aubert J, Gastine T, Fournier A (2017) Spherical convective dynamos in the rapidly rotating asymptotic regime. J Fluid Mech 813:558-593

Backus G, Gilbert F (1968) The resolving power of gross earth data. Geophys J Int 16(2):169-205

Backus G, Gilbert F (1970) Uniqueness in the inversion of inaccurate gross earth data. Philos Trans R Soc Lond A Math Phys Eng Sci 266(1173):123-192

Baerenzung J, Holschneider M, Wicht J, Sanchez S, Lesur V (2018) Modeling and predicting the short-term evolution of the geomagnetic field. J Geophys Res Solid Earth 123(6):4539-4560

Baerenzung J, Holschneider M, Wicht J, Lesur V, Sanchez S (2020) The Kalmag model as a candidate for IGRF-13. Earth Planets Space 72(1):163. https://doi.org/10.1186/s40623-020-01295-y

Barraclough D, Malin S (1979) Geomagnetic secular acceleration. Geophys J Int 58(3):785-793

Barrois O, Hammer MD, Finlay CC, Martin Y, Gillet N (2018) Assimilation of ground and satellite magnetic measurements: inference of core surface magnetic and velocity field changes. Geophys $J$ Int 215:695-712

Barton G, Barton G (1989) Elements of Greens functions and propagation: potentials, diffusion, and waves. Oxford University Press, Oxford

Beggan CD, Whaler KA, Macmillan S (2009) Biased residuals of core flow models from satellite-derived virtual observatories. Geophys J Int 177(2):463-475

Beggan CD, Saarimäki J, Whaler KA, Simons FJ (2013) Spectral and spatial decomposition of lithospheric magnetic field models using spherical Slepian functions. Geophys J Int 193(1):136-148

Bloxham J (1992) The steady part of the secular variation of the Earth's magnetic field. J Geophys Res Solid Earth 97(B13):19565-19579

Bloxham J, Jackson A (1992) Time-dependent mapping of the magnetic field at the core-mantle boundary. J Geophys Res Solid Earth 97(B13):19537-19563

Bouligand C, Gillet N, Jault D, Schaeffer N, Fournier A, Aubert J (2016) Frequency spectrum of the geomagnetic field harmonic coefficients from dynamo simulations. Geophys J Int 207(2):1142-1157

Braginsky SI (1970) Torsional magnetohydrodynamic vibrations in the Earth's core and variations in day length. Geomagn Aeron 10:1-8

Braginsky SI (1993) Mac-oscillations of the hidden ocean of the core. J Geomagn Geoelectr 45(11-12):1517-1538

Braginsky SI (1999) Dynamics of the stably stratified ocean at the top of the core. Phys Earth Planet Inter 111(1-2):21-34

Brown W, Mound J, Livermore P (2013) Jerks abound: an analysis of geomagnetic observatory data from 1957 to 2008. Phys Earth Planet Inter 223:62-76. https://doi.org/10.1016/j.pepi.2013.06.001

Buffett B, Matsui H (2015) A power spectrum for the geomagnetic dipole moment. Earth Planet Sci Lett 411:20-26

Buffett B, Matsui H (2019) Equatorially trapped waves in Earth's core. Geophys J Int 218(2):1210-1225

Cafarella L, DeSantis A, Meloni A (1992) Secular variation in Italy from historical geomagnetic field measurements. Phys Earth Planet Inter 73:206-221

Cain JC, Hendricks SJ, Langel RA, Hudson WV (1967) A proposed model for the international geomagnetic reference field-1965. J Geomagn Geoelectr 19(4):335-355

Chambodut A, Panet I, Mandea M, Diament M, Holschneider M, Jamet O (2005) Wavelet frames: an alternative to spherical harmonic representation of potential fields. Geophys J Int 163(3):875-899

Chao BF, Chung W, Shih Z, Hsieh Y (2014) Earth's rotation variations: a wavelet analysis. Terra Nova 26(4):260-264

Chi-Durán R, Avery MS, Knezek N, Buffett BA (2020) Decomposition of geomagnetic secular acceleration into traveling waves using complex empirical orthogonal functions. Geophys Res Lett 47(17):e2020GL087940

Christensen U, Wardinski I, Lesur V (2012) Timescales of geomagnetic secular acceleration in satellite field models and geodynamo models. Geophys J Int 190(1):243-254

Chulliat A, Maus S (2014) Geomagnetic secular acceleration, jerks, and a localized standing wave at the core surface from 2000 to 2010. J Geophys Res Solid Earth 119(3):1531-1543

Chulliat A, Alken P, Maus S (2015) Fast equatorial waves propagating at the top of the Earth's core. Geophys Res Lett 42(9):3321-3329

Constable C, Johnson C (2005) A paleomagnetic power spectrum. Phys Earth Planet Inter 153(1):61-73

Constable CG, Parker RL, Stark PB (1993) Geomagnetic field models incorporating frozen-flux constraints. Geophys J Int 113(2):419-433 
Courtillot V, Ducruix J, Le Mouël JL (1978) Sur une accélération récente de la variation séculaire du champ magnétique terrestre. C R Acad Sci Paris Ser D 287:1095-1098

Cox G, Livermore P, Mound J (2016) The observational signature of modelled torsional waves and comparison to geomagnetic jerks. Phys Earth Planet Inter 255:50-65. https://doi.org/10.1016/j.pepi.2016.03. 012

De Michelis P, Cafarella L, Meloni A (2000) A global analysis of the 1991 geomagnetic jerk. Geophys J Int 143(3):545-556. https://doi.org/10.1046/j.1365-246X.2000.00208.x

De Santis A, Barraclough D, Tozzi R (2003) Spatial and temporal spectra of the geomagnetic field and their scaling properties. Phys Earth Planet Inter 135(2):125-134

Domingos J, Pais MA, Jault D, Mandea M (2019) Temporal resolution of internal magnetic field modes from satellite data. Earth Planets Space 71(1):1-17

Finlay C, Dumberry M, Chulliat A, Pais M (2010a) Short timescale core dynamics: theory and observations. Space Sci Rev 155(1-4):177-218

Finlay C, Maus S, Beggan C, Hamoudi M, Lowes FJ, Olsen N, Thébault E (2010b) Evaluation of candidate geomagnetic field models for IGRF-11. Earth Planets Space 62(10):787-804. https://doi.org/10.5047/ eps.2010.11.005

Finlay CC, Olsen N, Kotsiaros S, Gillet N, Tøffner-Clausen L (2016) Recent geomagnetic secular variation from swarm and ground observatories as estimated in the CHAOS-6 geomagnetic field model. Earth Planets Space 68(1):1-18

Finlay C, Lesur V, Thébault E, Vervelidou F, Morschhauser A, Shore R (2017) Challenges handling magnetospheric and ionospheric signals in internal geomagnetic field modelling. Space Sci Rev 206(1-4):157-189

Finlay CC, Kloss C, Olsen N, Hammer MD, Tøffner-Clausen L, Grayver A, Kuvshinov A (2020) The CHAOS-7 geomagnetic field model and observed changes in the South Atlantic anomaly. Earth Planets Space 72(1):156. https://doi.org/10.1186/s40623-020-01252-9

Fisher R (1953) Dispersion on a sphere. Proc R Soc Lond A Math Phys Eng Sci R Soc 217(1130):295-305

Gastine T, Aubert J, Fournier A (2020) Dynamo-based limit to the extent of a stable layer atop Earth's core. Geophys J Int 222(2):1433-1448

Geese A, Mandea M, Lesur V, Hayn M (2010) Regional modelling of the Southern African geomagnetic field using harmonic splines. Geophys J Int 181(3):1329-1342

Gerick F, Jault D, Noir J (2020) Fast quasi-geostrophic magneto-coriolis modes in earth's core. Geophys Res Lett 48, E2020GL090803. https://doi.org/10.1029/2020GL090803

Gillet N (2019) Spatial and temporal changes of the geomagnetic field: insights from forward and inverse core field models. In: Mandea M, Korte M, Yau A, Petrovsky E (eds) Geomagnetism, Aeronomy and Space Weather: A Journey from the Earth's Core to the Sun (Special Publications of the International Union of Geodesy and Geophysics. Cambridge University Press, Cambridge, pp 115-132. https://doi.org/10.1017/9781108290135.010

Gillet N, Pais MA, Jault D (2009) Ensemble inversion of time-dependent core flow models. Geochem Geophys Geosyst 10(6). https://doi.org/10.1029/2008GC002290

Gillet N, Jault D, Canet E, Fournier A (2010) Fast torsional waves and strong magnetic field within the Earth's core. Nature 465(7294):74-77

Gillet N, Jault D, Finlay C, Olsen N (2013) Stochastic modelling of the Earth's magnetic field: inversion for covariances over the observatory era. Geochem Geophys Geosyst 14(4):766-786. https://doi. org/10.1002/ggge.2004441

Gillet N, Jault D, Finlay C (2015) Planetary gyre, time-dependent eddies, torsional waves, and equatorial jets at the Earth's core surface. J Geophys Res Solid Earth 120(6):3991-4013

Gillet N, Huder L, Aubert J (2019) A reduced stochastic model of core surface dynamics based on geodynamo simulations. Geophys J Int 219(1):522-539

Grayver AV, Olsen N (2019) The magnetic signatures of the $M_{2}, N_{2}$, and $O_{1}$ oceanic tides observed in swarm and champ satellite magnetic data. Geophys Res Lett 46(8):4230-4238. https://doi.org/10. 1029/2019GL082400

Gubbins D, Roberts N (1983) Use of the frozen flux approximation in the interpretation of archaeomagnetic and palaeomagnetic data. Geophys J Int 73(3):675-687

Haines G (1985) Spherical cap harmonic analysis. J Geophys Res Solid Earth 90(B3):2583-2591

Hammer MD, Finlay CC (2019) Local averages of the core-mantle boundary magnetic field from satellite observations. Geophys J Int 216(3):1901-1918

Hammer MD, Cox GA, Brown WJ, Beggan CD, Finlay CC (2021a) Geomagnetic Virtual Observatories: monitoring geomagnetic secular variation with the Swarm satellites. Earth Planets Space 73(1): $1-22$ 
Hammer MD, Finlay CC, Olsen N (2021b) Applications for CryoSat-2 satellite magnetic data in studies of Earth's core field variations. Earth Planets Space 73(1):73. https://doi.org/10.1186/ s40623-021-01365-9

Helffrich G, Kaneshima S (2010) Outer-core compositional stratification from observed core wave speed profiles. Nature 468(7325):807-810

Hide R (1966) Free hydromagnetic oscillations of the Earth's core and the theory of the geomagnetic secular variation. Philos Trans R Soc Lond A 259(1107):615-647

Hodder BM (1982) Monopoly. Geophys J Int 70(1):217-228

Holme R, De Viron O (2013) Characterization and implications of intradecadal variations in length of day. Nature 499(7457):202-204

Holschneider M, Chambodut A, Mandea M (2003) From global to regional analysis of the magnetic field on the sphere using wavelet frames. Phys Earth Planet Inter 135(2-3):107-124

Holschneider M, Lesur V, Mauerberger S, Baerenzung J (2016) Correlation based modelling and separation of geomagnetic field components. J Geophys Res Solid Earth 121:3142-3160. https://doi.org/ 10.1002/2015JB012629

Huder L, Gillet N, Thollard F (2019) Pygeost: a python package for geomagnetic data assimilation using stochastic processes. Geosci Model Dev 12:3795-3803. https://doi.org/10.5194/ gmd-12-3795-2019

Huder L, Gillet N, Finlay CC, Hammer MD, Tchoungui H (2020) COV-OBS.x2: 180 years of geomagnetic field evolution from ground-based and satellite observations. Earth Planets Space 72(1):1-18

Irving JC, Cottaar S, Lekić V (2018) Seismically determined elastic parameters for Earth's outer core. Sci Adv 4(6):eaar2538

Jackson A, Maffei S (2020) Plesio-geostrophy for Earth's core: I. basic equations, inertial modes and induction. Proc R Soc A 476(2243):20200513

Jackson A, Jonkers AR, Walker MR (2000) Four centuries of geomagnetic secular variation from historical records. Philos Trans R Soc Lond A Math Phys Eng Sci 358(1768):957-990

Jault D (2008) Axial invariance of rapidly varying diffusionless motions in the Earth's core interior. Phys Earth Planet Inter 166(1-2):67-76

Jault D (2015) Illuminating the electrical conductivity of the lowermost mantle from below. Geophys J Int 202:482-496

Jault D, Finlay CC (2015) Waves in the core and mechanical core-mantle interactions. In: Schubert G, Olson P (eds) Treatise on geophysics, core dynamics, chapter 8.09, vol 8, 2nd edn. Elsevier, Oxford, pp 225-244

Kloss C, Finlay CC (2019) Time-dependent low-latitude core flow and geomagnetic field acceleration pulses. Geophys J Int 217(1):140-168

Knezek N, Buffett B (2018) Influence of magnetic field configuration on magnetohydrodynamic waves in Earth's core. Phys Earth Planet Inter 277:1-9

Kono M (2007) Geomagnetism in perspective. In: Schubert G (ed) Treatise on Geophysics, Geomagnetism. Elsevier Science, Vol 5, pp 1-31

Konôpkovà Z, McWilliams RS, Gómez-Pérez N, Goncharov AF (2016) Direct measurement of thermal conductivity in solid iron at planetary core conditions. Nature 534:99-101

Korte M, Mandea M, Matzka J (2009) A historical declination curve for Munich from different data sources. Phys Earth Planet Inter 177(3):161-172. https://doi.org/10.1016/j.pepi.2009.08.005

Kuvshinov A, Grayver A, Tøffner-Clausen L, Olsen N (2021) Probing 3-D electrical conductivity of the mantle using 6 years of Swarm, CryoSat-2 and observatory magnetic data and exploiting matrix q-responses approach. Earth Planets Space 73(1):67. https://doi.org/10.1186/s40623-020-01341-9

Langel R, Estes R (1985a) Large-scale, near-field magnetic fields from external sources and the corresponding induced internal field. J Geophys Res Solid Earth 90(B3):2487-2494

Langel RA, Estes R (1985b) The near-Earth magnetic field at 1980 determined from Magsat data. J Geophys Res Solid Earth 90(B3):2495-2509

Langel R, Kerridge D, Arraclough D, Malin S (1986) Geomagnetic temporal change. J Geomagn Geoelectr 38(6):573-597

Le Bars M, Cébron D, Le Gal P (2015) Flows driven by libration, precession, and tides. Annu Rev Fluid Mech 47:163-193

Lesur V (2006) Introducing localized constraints in global geomagnetic field modelling. Earth Planets Space 58(4):477-483

Lesur V, Macmillan S, Thomson A (2005) The BGS magnetic field candidate models for the 10th generation IGRF. Earth Planets Space 57(12):1157-1163

Lesur V, Wardinski I, Rother M, Mandea M (2008) GRIMM: the GFZ reference internal magnetic model based on vector satellite and observatory data. Geophys J Int 173(2):382-394 
Lesur V, Wardinski I, Asari S, Minchev B, Mandea M (2010) Modelling the Earth's core magnetic field under flow constraints. Earth Planets Space 62:503-516. https://doi.org/10.5047/eps.2010.02.010

Lesur V, Rother M, Vervelidou F, Hamoudi M, Thébault E (2013) Post-processing scheme for modeling the lithospheric magnetic field. Solid Earth 4:105-118. https://doi.org/10.5194/sed-4-105-2013

Lesur V, Whaler K, Wardinski I (2015) Are geomagnetic data consistent with stably stratified flow at the core-mantle boundary? Geophys J Int 201(2):929-946. https://doi.org/10.1093/gji/ggv031

Lesur V, Wardinski I, Baerenzung J, Holschneider M (2018) On the frequency spectra of the core magnetic field Gauss coefficients. Phys Earth Planet Inter 276:145-158

Lhuillier F, Fournier A, Hulot G, Aubert J (2011) The geomagnetic secular-variation timescale in observations and numerical dynamo models. Geophys Res Lett 38:L09306. https://doi.org/10.1029/2011G L047356

Livermore PW, Hollerbach R, Finlay CC (2017) An accelerating high-latitude jet in Earth's core. Nat Geosci 10(1):62-68

Malin S (1969) Geomagnetic secular variation and its changes, 1942.5 to 1962.5. Geophys J Int 17(4):415-441

Malin SRC, Bullard E (1981) The direction of the Earth's magnetic field at London, 1570-1975. Philos Trans R Soc Lond 299:357-423

Malin SRC, Hodder BM (1982) Was the 1970 geomagnetic jerk of internal or external origin? Nature 296:726-728

Mandea M, Olsen N (2006) A new approach to directly determine the secular variation from magnetic satellite observations. Geophys Res Lett 33(15). https://doi.org/10.1029/2006GL026616

Mandea M, Holme R, Pais A, Pinheiro K, Jackson A, Verbanac G (2010) Geomagnetic jerks: rapid core field variations and core dynamics. Space Sci Rev 155(1-4):147-175

Masters G, Gubbins D (2003) On the resolution of density within the Earth. Phys Earth Planet Inter 140(1):159-167

Matzka J, Chulliat A, Mandea M, Finlay C, Qamili E (2010) Geomagnetic observations for main field studies: from ground to space. Space Sci Rev 155(1-4):29-64

Maus S, Macmillan S, Chernova T, Choi S, Dater D, Golovkov V, Lesur V, Lowes F, Lühr H et al (2005a) The 10th-generation international geomagnetic reference field. Geophys J Int 161(3):561-565

Maus S, Macmillan S, Lowes F, Bondar T (2005b) Evaluation of candidate geomagnetic field models for the 10th generation of IGRF. Earth Planets Space 57(12):1173-1181

Maus S, McLean S, Lühr H, Rother M, Mai W, Choi S (2005c) NGDC/GFZ candidate models for the 10th generation international geomagnetic reference field. Earth Planets Space 57(12):1151-1156

Mayhew M, Estes R (1983) Equivalent source modeling of the core magnetic field using Magsat data. J Geomagn Geoelectr 35(4):119-130

McLeod MG (1996) Spatial and temporal power spectra of the geomagnetic field. J Geophys Res 101:2745-2763

O’Brien MS, Parker RL (1994) Regularized geomagnetic field modelling using monopoles. Geophys J Int 118(3):566-578

Ohta K, Yagi T, Hirose K, Ohishi Y (2017) Thermal conductivity of ferropericlase in the Earth's lower mantle. Earth Planet Sci Lett 465:29-37

Olsen N, Mandea M (2007) Investigation of a secular variation impulse using satellite data: the 2003 geomagnetic jerk. Earth Planet Sci Lett 255(1):94-105

Olsen N, Mandea M (2008) Rapidly changing flows in the Earth's core. Nat Geosci 1(6):390-394

Olsen N, Stolle C (2012) Satellite geomagnetism. Annu Rev Earth Planet Sci 40:441-465

Olsen N, Lowes F, Sabaka TJ (2005a) Ionospheric and induced field leakage in geomagnetic field models, and derivation of candidate models for DGRF 1995 and DGRF 2000. Earth Planets Space 57(12):1191-1196

Olsen N, Sabaka TJ, Lowes F (2005b) New parameterization of external and induced fields in geomagnetic field modeling, and a candidate model for IGRF 2005. Earth Planets Space 57(12):1141-1149

Olsen N, Lühr H, Sabaka TJ, Mandea M, Rother M, Tøffner-Clausen L, Choi S (2006) CHAOS—a model of the Earth's magnetic field derived from CHAMP, ørsted, and SAC-C magnetic satellite data. Geophys J Int 166(1):67-75

Olsen N, Glassmeier KH, Jia X (2010) Separation of the magnetic field into external and internal parts. Space Sci Rev 152(1-4):135-157

Olsen N, Lühr H, Finlay CC, Sabaka TJ, Michaelis I, Rauberg J, Tøffner-Clausen L (2014) The CHAOS-4 geomagnetic field model. Geophys J Int 197(2):815-827

Olsen N, Albini G, Bouffard J, Parrinello T, Tøffner-Clausen L (2020) Magnetic observations from CryoSat-2: calibration and processing of satellite platform magnetometer data. Earth Planets Space 72:1-18 
Olson PL, Christensen UR, Driscoll PE (2012) From superchrons to secular variation: a broadband dynamo frequency spectrum for the geomagnetic dipole. Earth Planet Sci Lett 319:75-82

Pais M, Jault D (2008) Quasi-geostrophic flows responsible for the secular variation of the Earth's magnetic field. Geophys J Int 173(2):421-443

Pijpers FP, Thompson MJ (1994) The SOLA method for helioseismic inversion. Astron Astrophys 281:231-240

Plattner A, Simons F (2015) High-resolution local magnetic field models for the Martian South Pole from Mars Global Surveyor data. J Geophys Res Planets 120(9):1543-1566

Pozzo M, Davies C, Gubbins D, Alfe D (2012) Thermal and electrical conductivity of iron at Earth's core conditions. Nature 485(7398):355-358

Pujol JM (2013) The Backus-Gilbert method and their minimum-norm solution. Geophysics 78(3):W9-W30

Qamili E, De Santis A, Isac A, Mandea M, Duka B, Simonyan A (2013) Geomagnetic jerks as chaotic fluctuations of the Earth's magnetic field. Geochem Geophys Geosyst 14(4):839-850. https://doi.org/10. 1029/2012GC004398

Rogers HF, Beggan CD, Whaler KA (2019) Investigation of regional variation in core flow models using spherical Slepian functions. Earth Planets Space 71(1):19

Ropp G, Lesur V, Baerenzung J, Holschneider M (2020) Sequential modelling of the Earth's core magnetic field. Earth Planets Space 72(1):153. https://doi.org/10.1186/s40623-020-01230-1

Sabaka TJ, Langel RA, Baldwin RT, Conrad JA (1997) The geomagnetic field 1900-1995, including the large-scale field from magnetospheric sources, and the NASA candidate models for the 1995 revision of the IGRF. J Geomagn Geoelectr 49(2-3):157-206

Sabaka TJ, Tyler RH, Olsen N (2016) Extracting ocean-generated tidal magnetic signals from swarm data through satellite gradiometry. Geophys Res Lett 43(7):3237-3245. https://doi.org/10.1002/2016G L068180

Sabaka TJ, Tøffner-Clausen L, Olsen N, Finlay CC (2020) CM6: a comprehensive geomagnetic field model derived from both champ and swarm satellite observations. Earth Planets Space 72(1):80. https://doi. org/10.1186/s40623-020-01210-5

Sanchez S, Wicht J, Bärenzung J, Holschneider M (2019) Sequential assimilation of geomagnetic observations: perspectives for the reconstruction and prediction of core dynamics. Geophys $\mathrm{J}$ Int 217(2):1434-1450

Schaeffer N, Jault D, Nataf HC, Fournier A (2017) Turbulent geodynamo simulations: a leap towards Earth's core. Geophys J Int 211(1):1-29

Shore RM (2013) An improved description of Earth's external magnetic fields and their source regions using satellite data. PhD thesis, The University of Edinburgh

Shure L, Parker RL, Backus GE (1982) Harmonic splines for geomagnetic modelling. Phys Earth Planet Inter 28(3):215-229

Soloviev A, Chulliat A, Bogoutdinov S (2017) Detection of secular acceleration pulses from magnetic observatory data. Phys Earth Planet Inter 270:128-142

Thébault E, Schott J, Mandea M, Hoffbeck J (2004) A new proposal for spherical cap harmonic modelling. Geophys J Int 159(1):83-103

Thébault E, Finlay C, Alken P, Beggan C, Canet E, Chulliat A, Langlais B, Lesur V, Lowes F, Manoj C, Rother M, Schachtschneider R (2015) Evaluation of candidate geomagnetic field models for IGRF12. Earth Planets Space 67(112):1-23. https://doi.org/10.1186/s40623-015-0273-4

Verbanac G, Lühr H, Rother M, Korte M, Mandea M (2007) Contributions of the external field to the observatory annual means and a proposal for their corrections. Earth Planets Space. https://doi.org/10. 1186/BF03353102

Waddington R, Gubbins D, Barber N (1995) Geomagnetic field analysis-V. Determining steady core-surface flows directly from geomagnetic observations. Geophys J Int 122(1):326-350

Wardinski I, Holme R (2011) Signal from noise in geomagnetic field modelling: denoising data for secular variation studies. Geophys J Int 185(2):653-662

Whaler KA, Beggan CD (2015) Derivation and use of core surface flows for forecasting secular variation. J Geophys Res Solid Earth 120(3):1400-1414

Zhang K, Earnshaw P, Liao X, Busse F (2001) On inertial waves in a rotating fluid sphere. J Fluid Mech 437:103

Publisher's Note Springer Nature remains neutral with regard to jurisdictional claims in published maps and institutional affiliations. 